\title{
Synthesis and Antitubercular Activity of New L-serinyl Hydrazone Derivatives
}

Alessandra Campbell Pinheiro ${ }^{\mathrm{a}}$, Carlos Roland Kaiser ${ }^{\mathrm{b}}$, Thaís Cristina Mendonça Nogueira, ${ }^{\mathrm{a}, \mathrm{b}}$, Samir Aquino Carvalho $^{\mathrm{a}, \mathrm{b}}$, Edson Ferreira da Silva ${ }^{\mathrm{a}}$, Larisse de Oliveira Feitosa ${ }^{\mathrm{a}}$, Maria das Graças Müller de Oliveira Henriques $^{\mathrm{a}}$, André Luís Peixoto Candéa ${ }^{\mathrm{a}}$, Maria Cristina Silva Lourenço ${ }^{\mathrm{c}}$ and Marcus Vinícius Nora de Souza ${ }^{\mathrm{a}, \mathrm{b}_{*}}$

${ }^{a}$ Fundação Oswaldo Cruz, Instituto de Tecnologia em Fármacos-Far Manguinhos, Fundação Oswaldo Cruz, 21041250, Rio de Janeiro, RJ, Brazil

${ }^{b}$ Universidade Federal do Rio de Janeiro, Instituto de Química, Departamento de Química Orgânica, CP 68563, 21945 970- Rio de Janeiro, RJ, Brazil

${ }^{c}$ Fundação Oswaldo Cruz, Instituto de Pesquisas Clínicas Evandro Chagas, Departamento de Bacteriologia, Av. 21040 360, Rio de Janeiro, RJ, Brazil

\begin{abstract}
A series of 32 L-serinyl hydrazone derivatives have been synthesized and evaluated for their in vitro antibacterial activity against Mycobacterium tuberculosis $\mathrm{H}_{37} \mathrm{Rv}$, being also evaluated their cell viabilities in non infected and infected macrophages with Mycobacterium bovis Bacillus Calmette-Guerin (BCG). The compounds 8c, $8 \mathbf{e}, \mathbf{8 h}$ and $\mathbf{8 i}$, were non-cytotoxic and exhibited an important minimum inhibitory concentration (MIC) activity between 25 and $100 \mu \mathrm{g} / \mathrm{mL}$, which can be compared with that of the tuberculostatic drug D-cicloserine $(5-20 \mu \mathrm{g} / \mathrm{mL})$.
\end{abstract}

Keywords: BCG, D-cycloserine, hydrazone, L-cycloserine, L-serine, Mycobacterium, tuberculosis.

\section{INTRODUCTION}

Multidrug-resistant tuberculosis (MDR-TB) refers to organisms that are resistant to at least two of the first-line drugs, isoniazid and rifampin. There are approximately half a million new cases of MDR-TB worldwide every year [1]. More recently, extensively drug resistant tuberculosis (XDRTB) has been emerged. These bacteria are also resistant to three or more of the second-line treatment drugs. XDR-TB is considered a great public health problem, due to be resistant to the drugs routinely used to treat tuberculosis infections [25].

It is estimated that MDR-TB is responsible for about $3.3 \%$ of all new tuberculosis cases every year, and XDR-TB cases, extremely difficult to treat, have been confirmed in more than 58 countries [1]. Considering that, preventing MDR and XDR-TB from spreading is essential. Unfortunately, few treatment options have been available for XDR $\mathrm{TB}$, which are less effective when compared to traditional antibiotic therapies for TB.

Due to the high impact of MDR and XDR in TB treatment, new drugs and strategies to limit the spread of tuberculosis are much needed. In this context, the aim of this article is to describe the synthesis and biological activity of series of L-cicloserine analogs.

*Address correspondence to this author at the FioCruz - Fundação Oswaldo Cruz, Instituto de Tecnologia em Fármacos - Far Manguinhos, Rua Sizenando Nabuco, 100, Manguinhos, 21041-250 Rio de Janeiro, RJ, Brazil; Tel: +552139772404; Fax: +552125602518;

E-mail: marcos_souza@far.fiocruz.br
Our interest in the anti-TB profile of cicloserine analogs was supported by the structure of L-cicloserine 1a, featured in the natural product lactivicin $\mathbf{2}$, the first non- $\beta$-lactam broad-spectrum antibacterial agent with a mode of action similar to that of $\beta$-lactams [6] (Fig. 1).

L-cicloserine is also the $(S)$-enantiomer of D-cicloserine 1b, a broad-spectrum antibiotic classified as a second line drug for the treatment of tuberculosis. This drug inhibits $M$. tuberculosis in concentrations of 5 to $20 \mu \mathrm{g} / \mathrm{mL}$, with no cross-resistance observed (Fig. 2) [7-8].

In either the natural D- or synthetic L-isomer form, cicloserine acts as an essentially irreversible inhibitor of alanine racemase, responsible for the interconversion of alanine enantiomers, and thus represents the first step involved in bacterial cell wall biosynthesis [9-10].

Despite these findings, a very limited number of both Dand L-cycloserine analogs have been reported to date.
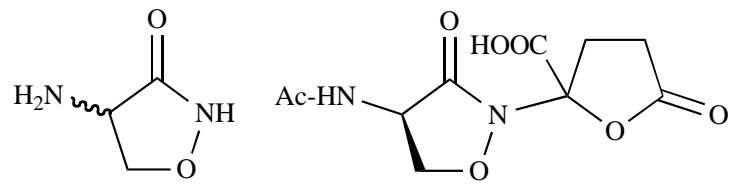

$$
\begin{aligned}
& \text { L-cycloserine, } \mathbf{1 a} \\
& \text { D-cycloserine, } \mathbf{1 b}
\end{aligned}
$$$$
\text { Lactivicin, } 2
$$

Fig. (1). D, L-cycloserine and the derivative Lactivicin. 
<smiles>N[C@@H]1CONC1=O</smiles>

L-cycloserine

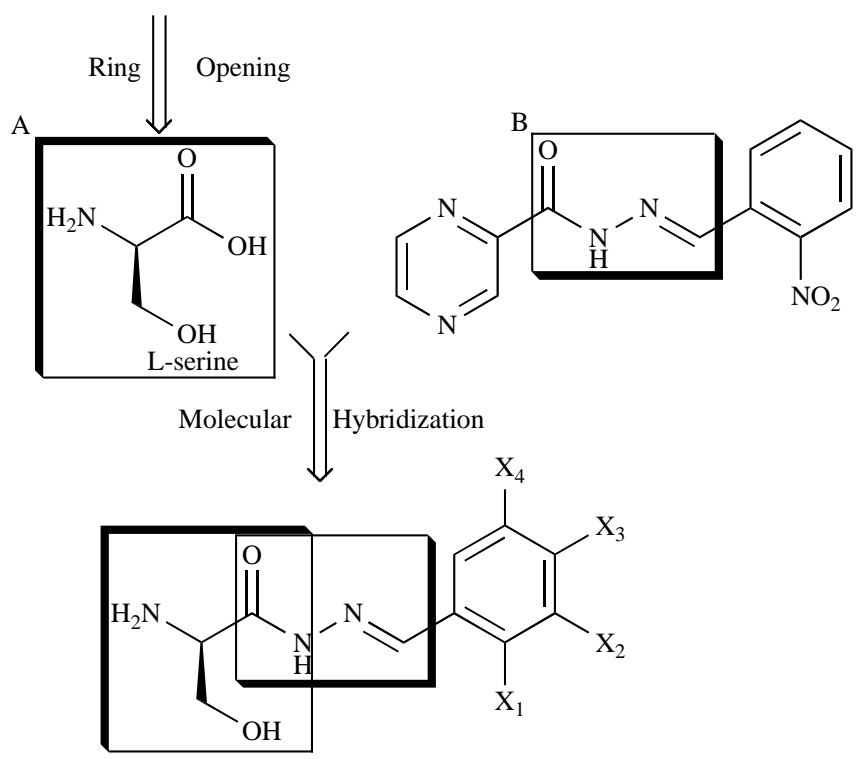

Fig. (2). Design concept of $N$-acylhydrazones, L-serine derivatives.

In our continuous search of new potent and safe antitubercular agents, we decided to synthesize a new class of Lserinyl hydrazone derivatives as attractive $\mathrm{L}$-cycloserine analogues, designed by molecular hybridization. $N$ acylhydrazones are described with a wide range of pharmacological activities, such as antibacterial agents [11-14]. For example, the pirazinamide derivative $\mathbf{B}$ prepared by our group, exhibiting a minimum inhibitory concentration (MIC) of $50 \mu \mathrm{g} / \mathrm{mL}$, which is four times more potent than the first line drug pyrazinamide [15].

The design concept of these compounds attempts to introduce the pharmacophoric $N$-acylhydrazone subunit of $\mathbf{B}$ into the core structure of L-serine $\mathbf{A}$, an acyclic L-cicloserine analog (Fig. 2).

\section{RESULTS AND DISCUSSION}

\section{Chemistry}

The synthetic route used for the preparation of the $(S)$ title compounds is outlined in the Scheme 1. L-serine amino acid was employed as starting material, and $t$-butoxycarbonyl (BOC) and benzyloxycarbonyl (Cbz) protecting groups were included in the core structure $\mathbf{2}$ due to an intrinsic instability observed in non-protected derivatives. This modification could also result in better pharmacodymamic profiles.

From L-serine, compounds 8 and $\mathbf{9}$ were obtained by esterification, leading $\mathbf{3}$, followed by $\mathrm{N}$-protection using $\mathrm{CbzCl}$ or $(\mathrm{BOC})_{2} \mathrm{O}$ in order to furnish $\mathbf{4}$ or $\mathbf{5}$ in 93 and $85 \%$ yield, respectively. These compounds were converted into their hydrazine derivatives using $80 \%$ aqueous hydrazine hydrate. After washing with cold ethanol the pure product 6 was obtained in $78 \%$ yield. In order to obtain 7 the crude product was column chromatographed on silica gel, affording the desired compound in $70 \%$ yield. Finally, after condensation reactions of these compounds with substituted aldehydes, the desired derivatives 8a-o and 9a-o were obtained in 70-89\% yield (Table 1-2).

All these compounds were identified by spectral data. The ${ }^{1} \mathrm{H}$ NMR spectra of the final compounds (8a-o and 9a-o) were consistent with two geometric isomers at $\mathrm{C}=\mathrm{N}$ bond level, or only one compound, exhibiting two different conformations, and getting different levels of stability. An additional NMR study was performed at room temperature and at $60^{\circ} \mathrm{C}$, using compound $\mathbf{8 b}$, randomized chosen. Both experiments resulted in very similar spectra, suggesting the existence of two geometric isomers $(E$ and $Z)$ of each 8a-o and 9a-o compound obtained. Comparing both spectra (Fig. 3 ) it is possible to observe a coalescence of labile NH protons uniquely.

However, only $E$-isomers $\mathbf{8 b}, \mathbf{8 n}$ and 80 have been confirmed by X-ray crystallography [16-18].

It's possible to note in NMR spectra nearly 1:1 isomeric proportion for non-hydroxyl/methoxyl substituted derivatives (8a-f and 9a-f). In other RMN spectra, relative to 8g-o and 9g-o compounds, mainly one compound has been formed, being defined as an $E$-isomer after X-ray crystallography results.

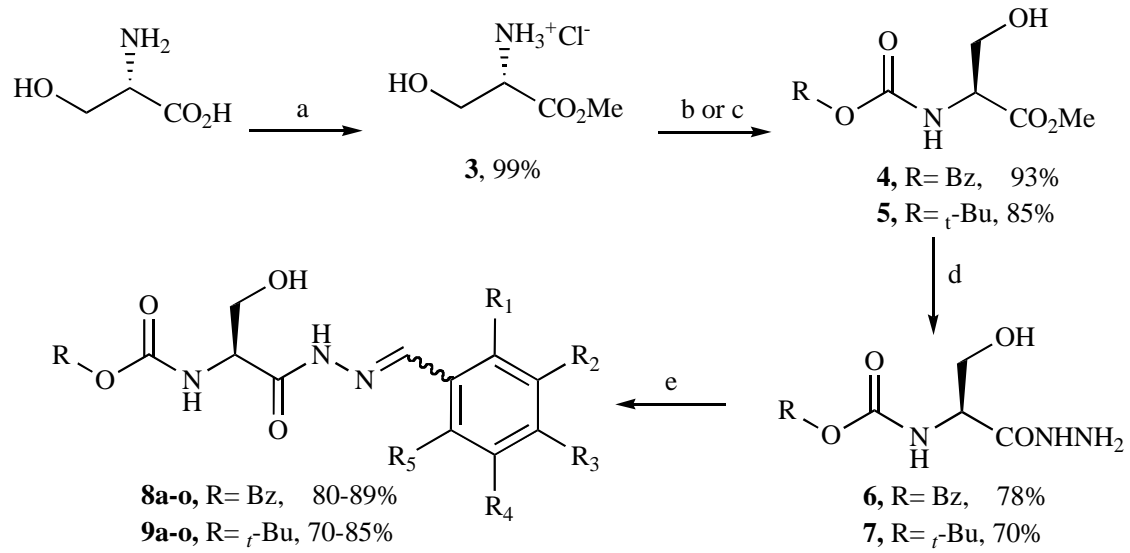

Scheme 1. Reagents and conditions: (a) $\mathrm{MeOH}, \mathrm{SOCl}_{2}$, rt, 24h; (b) $\mathrm{CbzCl}, \mathrm{NaHCO}_{3}, \mathrm{H}_{2} \mathrm{O}$, rt, 3h; (c) $(\mathrm{BOC})_{2} \mathrm{O}, \mathrm{Et}_{3} \mathrm{~N}, \mathrm{THF}, \mathrm{rt}, 24 \mathrm{~h}$; (d) $\mathrm{N}_{2} \mathrm{H}_{4} \cdot \mathrm{H}_{2} \mathrm{O}(80 \%), \mathrm{EtOH}, \mathrm{rt}, 24 \mathrm{~h}$; (e) EtOH, ArCHO, $80^{\circ} \mathrm{C}, 3-48 \mathrm{~h}$. 
Table 1. Physical and structural properties of benzyl (1S)-2-[2-(substituted-benzylidene)hydrazino]-1-(hydroxymethyl)-2oxoethylcarbamate (8a-o)

\begin{tabular}{|c|c|c|c|c|c|c|}
\hline \multirow{2}{*}{ Compound } & \multicolumn{5}{|c|}{ Substituents } & \multirow{2}{*}{ Yield (\%) } \\
\hline & $\mathbf{R}_{1}$ & $\mathbf{R}_{2}$ & $\mathbf{R}_{3}$ & $\mathbf{R}_{4}$ & $\mathbf{R}_{5}$ & \\
\hline $8 \mathbf{8}$ & $\mathrm{H}$ & $\mathrm{H}$ & $\mathrm{H}$ & $\mathrm{H}$ & $\mathrm{H}$ & 85 \\
\hline $8 \mathbf{b}$ & $\mathrm{NO}_{2}$ & $\mathrm{H}$ & $\mathrm{H}$ & $\mathrm{H}$ & $\mathrm{H}$ & 80 \\
\hline $8 c$ & $\mathrm{H}$ & $\mathrm{NO}_{2}$ & $\mathrm{H}$ & $\mathrm{H}$ & $\mathrm{H}$ & 80 \\
\hline $8 d$ & $\mathrm{H}$ & $\mathrm{H}$ & $\mathrm{NO}_{2}$ & $\mathrm{H}$ & $\mathrm{H}$ & 82 \\
\hline $8 e$ & $\mathrm{H}$ & $\mathrm{CN}$ & $\mathrm{H}$ & $\mathrm{H}$ & $\mathrm{H}$ & 82 \\
\hline $8 f$ & $\mathrm{H}$ & $\mathrm{H}$ & $\mathrm{CN}$ & $\mathrm{H}$ & $\mathrm{H}$ & 80 \\
\hline $8 g$ & $\mathrm{OH}$ & $\mathrm{H}$ & $\mathrm{H}$ & $\mathrm{H}$ & $\mathrm{H}$ & 89 \\
\hline $8 \mathrm{~h}$ & $\mathrm{OH}$ & $\mathrm{OH}$ & $\mathrm{H}$ & $\mathrm{H}$ & $\mathrm{H}$ & 81 \\
\hline $8 \mathbf{i}$ & $\mathrm{OH}$ & $\mathrm{H}$ & $\mathrm{OH}$ & $\mathrm{H}$ & $\mathrm{H}$ & 85 \\
\hline $8 \mathbf{j}$ & $\mathrm{OH}$ & $\mathrm{H}$ & $\mathrm{H}$ & $\mathrm{OH}$ & $\mathrm{H}$ & 87 \\
\hline $8 \mathbf{k}$ & $\mathrm{OH}$ & $\mathrm{OCH}_{3}$ & $\mathrm{H}$ & $\mathrm{H}$ & $\mathrm{H}$ & 80 \\
\hline 81 & $\mathrm{H}$ & $\mathrm{OH}$ & $\mathrm{OH}$ & $\mathrm{H}$ & $\mathrm{H}$ & 80 \\
\hline $8 m$ & $\mathrm{H}$ & $\mathrm{OCH}_{3}$ & $\mathrm{OCH}_{3}$ & $\mathrm{H}$ & $\mathrm{H}$ & 83 \\
\hline $8 n$ & $\mathrm{OH}$ & $\mathrm{H}$ & $\mathrm{OCH}_{3}$ & $\mathrm{H}$ & $\mathrm{H}$ & 80 \\
\hline 80 & $\mathrm{OCH}_{3}$ & $\mathrm{H}$ & $\mathrm{H}$ & $\mathrm{H}$ & $\mathrm{H}$ & 80 \\
\hline
\end{tabular}

Table 2. Physical and structural properties of $t$-Butyl (1S)-2-[2-(substituted-benzylidene)hydrazino]-1-(hydroxymethyl)-2oxoethylcarbamate (9a-o)

\begin{tabular}{|c|c|c|c|c|c|c|}
\hline \multirow{2}{*}{ Compound } & \multicolumn{5}{|c|}{ Substituents } & \multirow{2}{*}{ Yield (\%) } \\
\hline & $\mathbf{R}_{1}$ & $\mathbf{R}_{2}$ & $\mathbf{R}_{3}$ & $\mathbf{R}_{4}$ & $\mathbf{R}_{5}$ & \\
\hline $9 a$ & $\mathrm{H}$ & $\mathrm{H}$ & $\mathrm{H}$ & $\mathrm{H}$ & $\mathrm{H}$ & 82 \\
\hline $9 b$ & $\mathrm{NO}_{2}$ & $\mathrm{H}$ & $\mathrm{H}$ & $\mathrm{H}$ & $\mathrm{H}$ & 85 \\
\hline $9 \mathrm{c}$ & $\mathrm{H}$ & $\mathrm{NO}_{2}$ & $\mathrm{H}$ & $\mathrm{H}$ & $\mathrm{H}$ & 78 \\
\hline 9d & $\mathrm{H}$ & $\mathrm{H}$ & $\mathrm{NO}_{2}$ & $\mathrm{H}$ & $\mathrm{H}$ & 80 \\
\hline $9 e$ & $\mathrm{H}$ & $\mathrm{CN}$ & $\mathrm{H}$ & $\mathrm{H}$ & $\mathrm{H}$ & 78 \\
\hline 9f & $\mathrm{H}$ & $\mathrm{H}$ & $\mathrm{CN}$ & $\mathrm{H}$ & $\mathrm{H}$ & 70 \\
\hline $9 \mathrm{~g}$ & $\mathrm{OH}$ & $\mathrm{H}$ & $\mathrm{H}$ & $\mathrm{H}$ & $\mathrm{H}$ & 76 \\
\hline $9 \mathrm{~h}$ & $\mathrm{OH}$ & $\mathrm{OH}$ & $\mathrm{H}$ & $\mathrm{H}$ & $\mathrm{H}$ & 85 \\
\hline $9 \mathrm{i}$ & $\mathrm{OH}$ & $\mathrm{H}$ & $\mathrm{OH}$ & $\mathrm{H}$ & $\mathrm{H}$ & 74 \\
\hline $9 \mathbf{j}$ & $\mathrm{OH}$ & $\mathrm{H}$ & $\mathrm{H}$ & $\mathrm{OH}$ & $\mathrm{H}$ & 79 \\
\hline $9 k$ & $\mathrm{OH}$ & $\mathrm{OCH}_{3}$ & $\mathrm{H}$ & $\mathrm{H}$ & $\mathrm{H}$ & 80 \\
\hline 91 & $\mathrm{H}$ & $\mathrm{OH}$ & $\mathrm{OH}$ & $\mathrm{H}$ & $\mathrm{H}$ & 85 \\
\hline $9 \mathrm{~m}$ & $\mathrm{H}$ & $\mathrm{OCH}_{3}$ & $\mathrm{OCH}_{3}$ & $\mathrm{H}$ & $\mathrm{H}$ & 82 \\
\hline $9 n$ & $\mathrm{OH}$ & $\mathrm{H}$ & $\mathrm{OCH}_{3}$ & $\mathrm{H}$ & $\mathrm{H}$ & 84 \\
\hline 90 & $\mathrm{OCH}_{3}$ & $\mathrm{H}$ & $\mathrm{H}$ & $\mathrm{H}$ & $\mathrm{H}$ & 75 \\
\hline
\end{tabular}




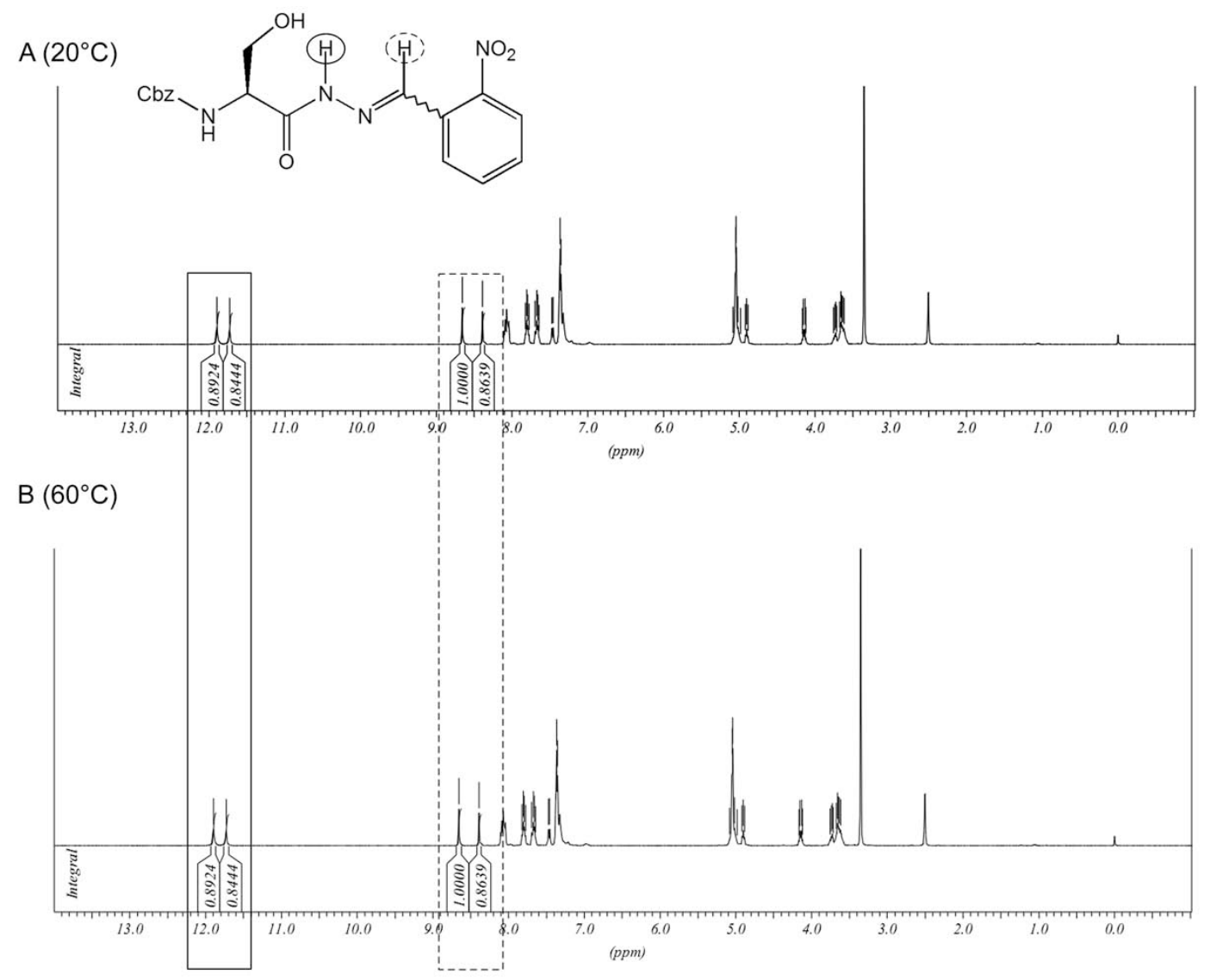

Fig. (3). NMR spectra of $8 \mathbf{b}$ at $20^{\circ} \mathrm{C}$ (A) and at $60^{\circ} \mathrm{C}(\mathrm{B})$. The labile $E$ and $Z$ protons of NHN subunit are continued line marked; not labile $E$ and $Z$ protons, exemplified by $\mathrm{N}=\mathrm{CH}$, are broken line marked.

\section{Antimycobacterial Activity}

The cellular viability of all synthesized compounds was previously evaluated by Mosman's MTT microcultured tetrazolium assay, and all compounds were considered not cytotoxic to the host cells, since they did not kill more than $10 \%$ of the cells at the concentration tested $(50 \mu \mathrm{g} / \mathrm{mL})$.

The antimycobacterial activities of all synthesized compounds were assessed against M. tuberculosis ATTC 27294 using the Microplate Alamar Blue Assay (MABA) (Tables 3-5). This methodology is nontoxic, uses thermally stable reagent, and shows good correlation with proportional and BACTEC radiometric methods.

Firstly, for the $N$-benzyloxycarbonyl-L-serinylhydrazone (8a-o) derivatives it was observed that the most active compound was 8c $(\mathrm{MIC}=25 \mu \mathrm{g} / \mathrm{mL})$, which included a nitro group in the meta position of the aromatic hydrazone subunit. This activity can be compared to the in vitro activity of D-cicloserine (MIC=5-20 $\mu \mathrm{g} / \mathrm{mL}$ ).

The antimycobacterial activity decreases when the nitro group of $\mathbf{8 c}$ is replaced by the cyano group (8e, $\mathrm{MIC}=50 \mu \mathrm{g} / \mathrm{mL}$ ), indicating the importance of a nitro group in this position for monosubstituted derivatives.

The presence of hydroxyl groups in orto position of the aromatic ring seems to indicate its relevance to the biological activity in the disubstituted derivatives $8 \mathbf{h}(\mathrm{MIC}=50 \mu \mathrm{g} / \mathrm{mL})$, $\mathbf{8 i}(\mathrm{MIC}=100 \mu \mathrm{g} / \mathrm{mL})$ and $\mathbf{8 k}(\mathrm{MIC}=100 \mu \mathrm{g} / \mathrm{mL})($ Table 3).
In order to evaluate the contribution of benzyloxycarbonyl protecting group $(\mathrm{Cbz})$ to the pharmacophoric character of these compounds to the action over M. tuberculosis, we performed the investigation of the tuberculostatic profile of $N$-t-butyloxycarbonyl-L-serinylhydrazone (9a-o) analogues. It was observed that the presence of $\mathrm{Cbz}$ in the structure of 8a-o seems to be important to the biological activity of this class of compounds, since compounds of 9a-o series did not remain active against $M$. tuberculosis (Table 4).

Furthermore, compounds 10a and 10b were synthesized from the $N$-Cbz-protected derivatives $8 \mathbf{c}$ and $\mathbf{8 e}$, respectively, with the aim of confirm the importance of $\mathrm{Cbz}$ and the oxazolidinone nucleus for the biological activity of these derivatives. The compounds 10a and 10b obtained, after a standard cyclization reaction using $\mathrm{NaH}$ in room temperature, showed decreased activity related to its starting materials (Table 5).

Also, analogous non-protected compound 11a is not active against $M$. tuberculosis, ratifying the importance of $\mathrm{Cbz}$ protecting group to the biological activity observed to the most active compounds synthesized, 8c (Fig. 4).

The biological activity observed for $N$-Cbz protected-Lserinylhydrazone derivatives could be related to $\operatorname{cog} P$ values obtained to these derivatives, indicating that lipophilicity is an important parameter for anti-TB activity in these series. 


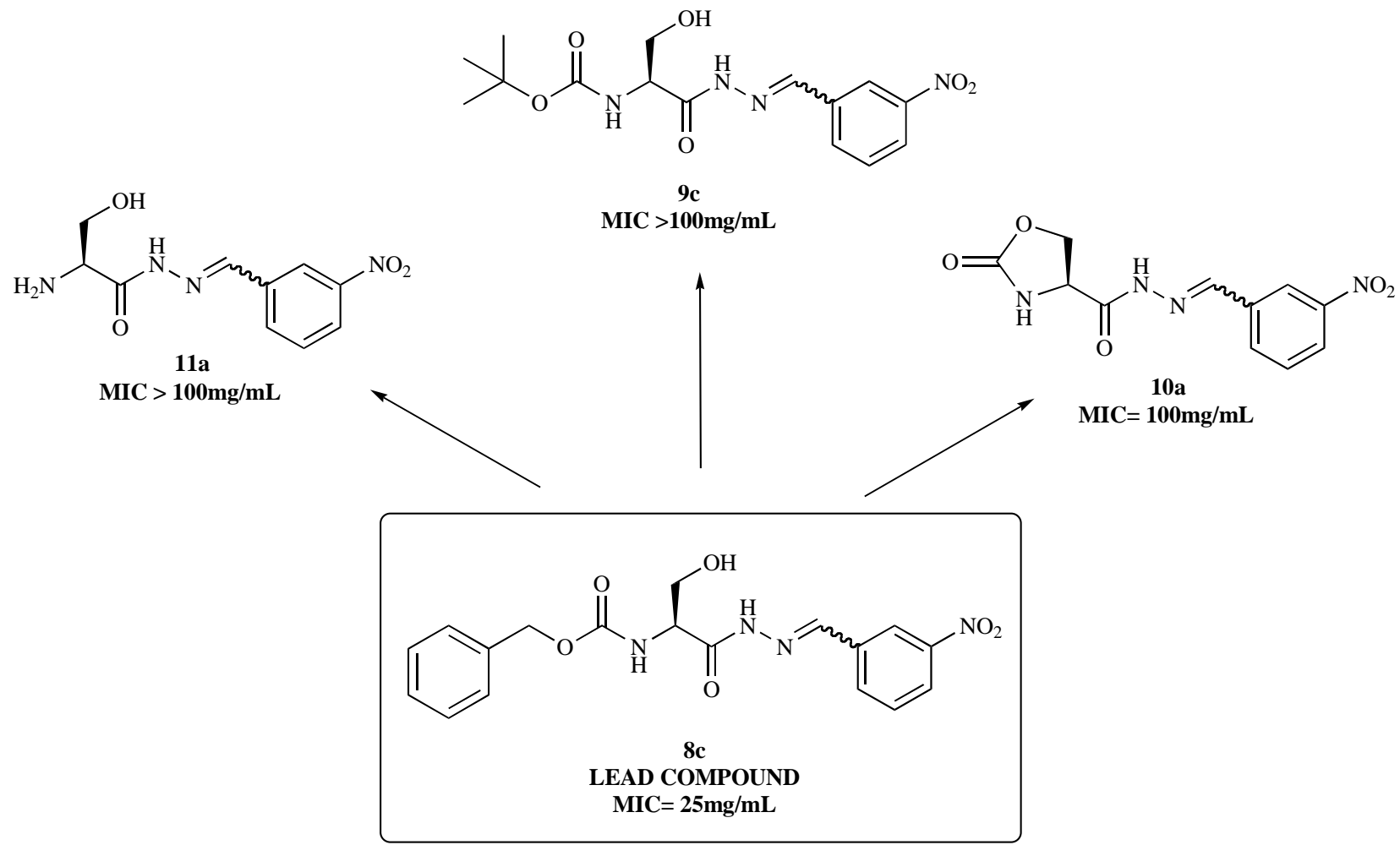

D-cycloserine: $\mathrm{MIC}=5-20 \mathrm{mg} / \mathrm{mL}$

Fig. (4). Biological activities of $8 \mathrm{c}$ and its analogous compounds 9c, 10a and 11a.

Table 3. The in vitro activity of compounds 8a-o against $M$. tuberculosis H37Rv strain (ATCC 27294, susceptible both to rifampin and isoniazid) and $\operatorname{cog} P$ calculations

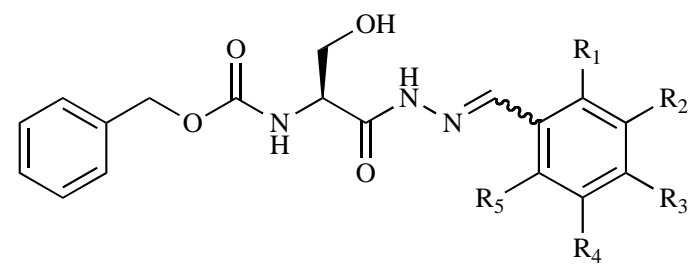

\begin{tabular}{|c|c|c|c|c|c|c|c|}
\hline \multirow{2}{*}{ Compound } & \multicolumn{5}{|c|}{ Substituents } & \multirow{2}{*}{$\begin{array}{c}\mathrm{MIC}^{\mathrm{a}} \\
(\mu \mathrm{g} / \mathrm{mL})\end{array}$} & \multirow{2}{*}{$\operatorname{clog} P^{\mathrm{h}}$} \\
\hline & $\mathbf{R}_{1}$ & $\mathbf{R}_{2}$ & $\mathbf{R}_{3}$ & $\mathbf{R}_{4}$ & $\mathbf{R}_{5}$ & & \\
\hline $8 a$ & $\mathrm{H}$ & $\mathrm{H}$ & $\mathrm{H}$ & $\mathrm{H}$ & $\mathrm{H}$ & $>100$ & 3.770 \\
\hline $8 \mathbf{b}$ & $\mathrm{NO}_{2}$ & $\mathrm{H}$ & $\mathrm{H}$ & $\mathrm{H}$ & $\mathrm{H}$ & $>100$ & 3.681 \\
\hline $8 c$ & $\mathrm{H}$ & $\mathrm{NO}_{2}$ & $\mathrm{H}$ & $\mathrm{H}$ & $\mathrm{H}$ & 25 & 3.705 \\
\hline $8 d$ & $\mathrm{H}$ & $\mathrm{H}$ & $\mathrm{NO}_{2}$ & $\mathrm{H}$ & $\mathrm{H}$ & $>100$ & 3.729 \\
\hline $8 e$ & $\mathrm{H}$ & $\mathrm{CN}$ & $\mathrm{H}$ & $\mathrm{H}$ & $\mathrm{H}$ & 50 & 3.501 \\
\hline $8 f$ & $\mathrm{H}$ & $\mathrm{H}$ & $\mathrm{CN}$ & $\mathrm{H}$ & $\mathrm{H}$ & $>100$ & 3.525 \\
\hline $8 g$ & $\mathrm{OH}$ & $\mathrm{H}$ & $\mathrm{H}$ & $\mathrm{H}$ & $\mathrm{H}$ & $>100$ & 3.711 \\
\hline $8 \mathbf{h}$ & $\mathrm{OH}$ & $\mathrm{OH}$ & $\mathrm{H}$ & $\mathrm{H}$ & $\mathrm{H}$ & 50 & 3.007 \\
\hline $8 i$ & $\mathrm{OH}$ & $\mathrm{H}$ & $\mathrm{OH}$ & $\mathrm{H}$ & $\mathrm{H}$ & 100 & 3.207 \\
\hline $8 \mathbf{j}$ & $\mathrm{OH}$ & $\mathrm{H}$ & $\mathrm{H}$ & $\mathrm{OH}$ & $\mathrm{H}$ & $>100$ & 3.207 \\
\hline $8 k$ & $\mathrm{OH}$ & $\mathrm{OCH}_{3}$ & $\mathrm{H}$ & $\mathrm{H}$ & $\mathrm{H}$ & 100 & 3.314 \\
\hline 81 & $\mathrm{H}$ & $\mathrm{OH}$ & $\mathrm{OH}$ & $\mathrm{H}$ & $\mathrm{H}$ & $>100$ & 2.802 \\
\hline
\end{tabular}


Table 3. contd....

\begin{tabular}{|c|c|c|c|c|c|c|c|}
\hline \multirow{2}{*}{ Compound } & \multicolumn{5}{|c|}{ Substituents } & \multirow{2}{*}{$\begin{array}{c}\mathrm{MIC}^{\mathrm{a}} \\
(\mu \mathrm{g} / \mathrm{mL})\end{array}$} & \multirow{2}{*}{$\operatorname{clog} P^{b}$} \\
\hline & $\mathbf{R}_{1}$ & $\mathbf{R}_{2}$ & $\mathbf{R}_{3}$ & $\mathbf{R}_{4}$ & $\mathbf{R}_{5}$ & & \\
\hline $8 m$ & $\mathrm{H}$ & $\mathrm{OCH}_{3}$ & $\mathrm{OCH}_{3}$ & $\mathrm{H}$ & $\mathrm{H}$ & $>100$ & 3.417 \\
\hline $8 n$ & $\mathrm{OH}$ & $\mathrm{H}$ & $\mathrm{OCH}_{3}$ & $\mathrm{H}$ & $\mathrm{H}$ & $>100$ & 3.743 \\
\hline 80 & $\mathrm{OCH}_{3}$ & $\mathrm{H}$ & $\mathrm{H}$ & $\mathrm{H}$ & $\mathrm{H}$ & $>100$ & 3.779 \\
\hline
\end{tabular}

${ }^{\mathrm{a}}$ Minimum inhibitory concentration

${ }^{\mathrm{b}}$ Calculated using online www.molinspiration.com website

Table 4. The in vitro activity of compounds 9a-o against $M$. tuberculosis H37Rv strain (ATCC 27294, susceptible both to rifampin and isoniazid) and $\operatorname{cog} P$ calculations<smiles>[R]c1c([R])c([R])c(/C=N/NC(=O)[C@H](CO)NC(=O)OC(C)(C)C)c([R])c1[R]</smiles>

\begin{tabular}{|c|c|c|c|c|c|c|c|}
\hline \multirow{2}{*}{ Compound } & \multicolumn{5}{|c|}{ Substituents } & \multirow{2}{*}{$\begin{array}{c}\text { MIC }^{\mathrm{a}} \\
(\mu \mathrm{g} / \mathrm{mL})\end{array}$} & \multirow{2}{*}{$\operatorname{clog} P^{\mathrm{b}}$} \\
\hline & $\mathbf{R}_{1}$ & $\mathbf{R}_{\mathbf{2}}$ & $\mathbf{R}_{3}$ & $\mathbf{R}_{4}$ & $\mathbf{R}_{5}$ & & \\
\hline $9 a$ & $\mathrm{H}$ & $\mathrm{H}$ & $\mathrm{H}$ & $\mathrm{H}$ & $\mathrm{H}$ & $>100$ & 3.361 \\
\hline $9 b$ & $\mathrm{NO}_{2}$ & $\mathrm{H}$ & $\mathrm{H}$ & $\mathrm{H}$ & $\mathrm{H}$ & $>100$ & 3.272 \\
\hline $9 \mathrm{c}$ & $\mathrm{H}$ & $\mathrm{NO}_{2}$ & $\mathrm{H}$ & $\mathrm{H}$ & $\mathrm{H}$ & $>100$ & 3.296 \\
\hline 9d & $\mathrm{H}$ & $\mathrm{H}$ & $\mathrm{NO}_{2}$ & $\mathrm{H}$ & $\mathrm{H}$ & $>100$ & 3.320 \\
\hline $9 e$ & $\mathrm{H}$ & $\mathrm{CN}$ & $\mathrm{H}$ & $\mathrm{H}$ & $\mathrm{H}$ & $>100$ & 3.092 \\
\hline $9 f$ & $\mathrm{H}$ & $\mathrm{H}$ & $\mathrm{CN}$ & $\mathrm{H}$ & $\mathrm{H}$ & $>100$ & 3.116 \\
\hline $9 \mathrm{~g}$ & $\mathrm{OH}$ & $\mathrm{H}$ & $\mathrm{H}$ & $\mathrm{H}$ & $\mathrm{H}$ & $>100$ & 3.301 \\
\hline $9 \mathrm{~h}$ & $\mathrm{OH}$ & $\mathrm{OH}$ & $\mathrm{H}$ & $\mathrm{H}$ & $\mathrm{H}$ & $>100$ & 2.598 \\
\hline $9 \mathbf{i}$ & $\mathrm{OH}$ & $\mathrm{H}$ & $\mathrm{OH}$ & $\mathrm{H}$ & $\mathrm{H}$ & $>100$ & 2.798 \\
\hline $9 j$ & $\mathrm{OH}$ & $\mathrm{H}$ & $\mathrm{H}$ & $\mathrm{OH}$ & $\mathrm{H}$ & $>100$ & 2.798 \\
\hline $9 k$ & $\mathrm{OH}$ & $\mathrm{OCH}_{3}$ & $\mathrm{H}$ & $\mathrm{H}$ & $\mathrm{H}$ & $>100$ & 2.905 \\
\hline 91 & $\mathrm{H}$ & $\mathrm{OH}$ & $\mathrm{OH}$ & $\mathrm{H}$ & $\mathrm{H}$ & $>100$ & 2.393 \\
\hline $9 m$ & $\mathrm{H}$ & $\mathrm{OCH}_{3}$ & $\mathrm{OCH}_{3}$ & $\mathrm{H}$ & $\mathrm{H}$ & $>100$ & 3.008 \\
\hline $9 n$ & $\mathrm{OH}$ & $\mathrm{H}$ & $\mathrm{OCH}_{3}$ & $\mathrm{H}$ & $\mathrm{H}$ & $>100$ & 3.334 \\
\hline 90 & $\mathrm{OCH}_{3}$ & $\mathrm{H}$ & $\mathrm{H}$ & $\mathrm{H}$ & $\mathrm{H}$ & $>100$ & 3.370 \\
\hline
\end{tabular}

${ }^{a}$ Minimum inhibitory concentration

${ }^{\mathrm{b}}$ Calculated using online www.molinspiration.com website

\section{Cell Viability Assay}

Cellular viability in the presence and absence of test compounds $(\mathbf{8 c}, 8 \mathbf{e}, \mathbf{8 h}, \mathbf{8 i}, \mathbf{8 k}$ and 10a) was determined by Mosman's MTT (3-(4,5-dimethylthiazol-2yl)-2,5-dimethyl tetrazolium bromide; Merck) microcultured tetrazolium assay. The results were represented as percentage cell viability (Table 6). This table shows that the compounds $8 \mathbf{c}, \mathbf{8 e}, \mathbf{8 h}$ and $\mathbf{8 i}$, with $100 \%$ of cell viability, were not toxic to the host cells in the minimum concentration tested. These compounds were selected to be tested in macrophages infected with BCG (Table 7).

This test is able to evaluate the action of these compounds over macrophages that exhibit a modified metabolism after infection. After this test the derivatives $\mathbf{8 c}, \mathbf{8 e}, \mathbf{8 h}$ and $8 \mathbf{i}$ were not cytotoxic, due to they did not kill more than $10 \%$ of the cells at the maximum concentration tested. 
Table 5. The in vitro activity of compounds 10a-b and 11a against M. tuberculosis H37Rv strain (ATCC 27294, susceptible both to rifampin and isoniazid) and $\operatorname{clog} P$ calculations<smiles>[R]C1=C([R])C([R])C(C=NNC(=O)[C@@H]2COC(=O)N2)=C([R])C1[R]</smiles>

\begin{tabular}{|c|c|c|c|c|c|c|c|}
\hline \multirow{2}{*}{ Compound } & \multicolumn{5}{|c|}{ Substituents } & \multirow{2}{*}{$\begin{array}{c}M^{M I C} C^{a} \\
(\mu \mathrm{g} / \mathrm{mL})\end{array}$} & \multirow{2}{*}{$\operatorname{clog} P^{h}$} \\
\hline & $\mathbf{R}_{1}$ & $\mathbf{R}_{2}$ & $\mathbf{R}_{3}$ & $\mathbf{R}_{4}$ & $\mathbf{R}_{5}$ & & \\
\hline $10 a$ & $\mathrm{H}$ & $\mathrm{NO}_{2}$ & $\mathrm{H}$ & $\mathrm{H}$ & $\mathrm{H}$ & 100 & 0.805 \\
\hline $10 \mathrm{~b}$ & $\mathrm{H}$ & $\mathrm{CN}$ & $\mathrm{H}$ & $\mathrm{H}$ & $\mathrm{H}$ & $>100$ & 0.601 \\
\hline $11 a$ & $\mathrm{H}$ & $\mathrm{NO}_{2}$ & $\mathrm{H}$ & $\mathrm{H}$ & $\mathrm{H}$ & $>100$ & -0.447 \\
\hline
\end{tabular}

${ }^{\mathrm{a}}$ Minimum inhibitory concentration

${ }^{\mathrm{b}}$ Calculated using online www.molinspiration.com website

Table 6. Data of the cellular viability for a macrophage cell line J774 (ATCC TIB-67 ${ }^{\text {TM }}$ ) by Mosman's assay

\begin{tabular}{|c|c|c|c|}
\hline Compound & $\mathbf{1 0} \boldsymbol{\mu g} / \mathbf{m L}$ & $\mathbf{5 0} \boldsymbol{\mu g} / \mathbf{m L}$ & $\mathbf{1 0 0} \boldsymbol{\mu g} / \mathbf{m L}$ \\
\hline \hline $\mathbf{8 c}$ & $100 \%$ & $100 \%$ & $100 \%$ \\
\hline $\mathbf{8 e}$ & $100 \%$ & $100 \%$ & $100 \%$ \\
\hline $\mathbf{8 h}$ & $100 \%$ & $100 \%$ & $100 \%$ \\
\hline $\mathbf{8 i}$ & $100 \%$ & $100 \%$ & $100 \%$ \\
\hline $\mathbf{8 k}$ & $91 \%$ & $91 \%$ & $78 \%$ \\
\hline $\mathbf{1 0 a}$ & $93 \%$ & $93 \%$ & $91 \%$ \\
\hline
\end{tabular}

Table 7. Data of the cellular viability for a macrophage cell line J774 (ATCC TIB-67 ${ }^{\text {TM}}$ ) infected with BCG by Mosman's assay

\begin{tabular}{|c|c|c|c|}
\hline Compound & $\mathbf{1 0} \boldsymbol{\mu g} / \mathbf{m L}$ & $\mathbf{5 0} \boldsymbol{\mu g} / \mathbf{m L}$ & $\mathbf{1 0 0} \boldsymbol{\mu g} / \mathbf{m L}$ \\
\hline \hline $\mathbf{8 c}$ & $100 \%$ & $100 \%$ & $100 \%$ \\
\hline $\mathbf{8 e}$ & $100 \%$ & $100 \%$ & $96 \%$ \\
\hline $\mathbf{8 h}$ & $100 \%$ & $100 \%$ & $100 \%$ \\
\hline $\mathbf{8 i}$ & $100 \%$ & $100 \%$ & $100 \%$ \\
\hline
\end{tabular}

\section{CONCLUSION}

The synthesis of thirty-two compounds, including thirty benzyl/t-butyl (1S)-2-[2-(substituted-benzylidene)hydrazino] -1-(hydroxymethyl)-2-oxoethylcarbamate (8a-o, 9a-o) and two (4S)- $N$ '-(substituted-phenyl)methylidene)-2-oxo-1,3oxazolidine-4-carbohydrazide (10a-b) was performed in good yields (50-89\%). Also, 30 new compounds (8b-c, 8e-o, 9a-o and 10a-b) were identified.

All of these compounds were submitted to antimycobacterial activity evaluation. The compounds $8 \mathbf{c}, \mathbf{8 e}, \mathbf{8 h}, \mathbf{8 i}, \mathbf{8 k}$ and 10a exhibited activity between 25 and $100 \mu \mathrm{g} / \mathrm{mL}$, being compared to the tuberculostatic drug D-cicloserine (5$20 \mu \mathrm{g} / \mathrm{mL}$ ). After cell viability evaluation, it was found that $\mathbf{8 c}, \mathbf{8 e}, \mathbf{8 h}$ and $\mathbf{8 i}$ were not cytotoxic in non infected or infected macrophages with M. bovis Bacillus Calmette-Guerin (BCG). Further X-ray crystallography studies are under way in our laboratory.

\section{MATERIALS AND METHODS}

General Procedures. Melting points were determined on a Buchi apparatus and are uncorrected. Infrared spectra were recorded on a Thermo Nicolet Nexus 670 spectrometer as potassium bromide pellets and frequencies are expressed in $\mathrm{cm}^{-1}$. Mass spectra (ESI assay in solution of ammonium chloride) were recorded on Micromass ZQ Waters mass spectrometer. NMR spectra were recorded on a Bruker Avance 400 operating at $400.00 \mathrm{MHz}\left({ }^{1} \mathrm{H}\right)$ and $100.0 \mathrm{MHz}$ $\left({ }^{13} \mathrm{C}\right)$ and Bruker Avance 500 spectrometer operating at $500.00 \mathrm{MHz}\left({ }^{1} \mathrm{H}\right)$ and $125.0 \mathrm{MHz}\left({ }^{13} \mathrm{C}\right)$, in deuterated dimethylsulfoxide. Chemical shifts are reported in $\mathrm{ppm}(\delta)$ relative to tetramethylsilane and $J$-coupling in $\operatorname{Hertz}(\mathrm{Hz})$. Proton and carbon spectra were typically obtained at room temperature.

Synthesis of methyl (2S)-2-amino-3-hydroxypropanoate hydrochloride 3

To a stirred solution of thionyl chloride $(69.5 \mathrm{~mL}, 0.95$ mol) in methanol $(400 \mathrm{~mL})$ at $0^{0} \mathrm{C}$ was added $\mathrm{L}$-serine $(20 \mathrm{~g}$, $0.19 \mathrm{~mol}$ ), and the reaction mixture was stirred for $24 \mathrm{~h}$ at room temperature. The reaction mixture was concentrated under reduced pressure to give $3(147.2 \mathrm{~g})$ in quantitative yield.

Synthesis of methyl (2S)-2-[(benzyloxycarbonyl)amino]3-hydroxypropanoate 4

To a reaction mixture containing (2S)-2-amino-3hydroxypropanoate hydrochloride 3 (13.2 g, $0.11 \mathrm{~mol})$, water $(100 \mathrm{~mL})$, diethyl ether $(75 \mathrm{~mL})$ and sodium bicarbonate $(48 \mathrm{~g}, 0.55 \mathrm{~mol})$ at $0^{\circ} \mathrm{C}$ was added dropwise benzyl chloroformate $(21 \mathrm{~mL}, 0.15 \mathrm{~mol})$. After $2 \mathrm{~h}$ at $0^{\circ} \mathrm{C}$ and $1 \mathrm{~h}$ at room 
temperature, the reaction mixture was quenched with pyridine $(20 \mathrm{~mL})$, and water was added $(30 \mathrm{~mL})$. The organic layer was washed with $\mathrm{HCl}(2.5 \mathrm{~N}, 20 \mathrm{~mL})$, dried over anhydrous sodium sulfate and concentrated under reduced pressure. The residue was chromatographed using a gradient 10 to $30 \%$ ethylacetate in hexane, affording $\mathbf{4}$ as colorless oil in $80 \%$ yield.

Synthesis of methyl (2S)-2-[(tert-butoxycarbonyl)amino] -3-hydroxypropanoate 5

To a reaction mixture containing (2S)-2-amino-3hydroxypropanoate hydrochloride 3 ( $3 \mathrm{~g}, 19.3 \mathrm{mmol})$ in anhydrous THF $(40 \mathrm{~mL})$ at room temperature was added triethylamine $(3.2 \mathrm{~mL}, 23.2 \mathrm{mmol})$ and $(\mathrm{BOC})_{2} \mathrm{O}(6.32 \mathrm{~g}, 29$ mmol). The reaction mixture was stirred for 24 hours at room temperature, quenched with water $(40 \mathrm{~mL})$ and extracted with ethyl acetate. The combined organic phases were dried over anhydrous sodium sulfate and concentrated under reduced pressure. The residue was purified by column chromatography on silica gel using a gradient 0 to $50 \%$ ethylacetate in hexane, affording $\mathbf{5}$ as a colorless oil in $85 \%$ yield.

Synthesis of benzyl (1S)-2-hydrazino-1-(hydroxymethyl) -2-oxoethylcarbamate 6

To a stirred solution of $4(0.3 \mathrm{~g}, 1.17 \mathrm{mmol})$ in ethanol (10 mL) was added $\mathrm{N}_{2} \mathrm{H}_{4} \cdot \mathrm{H}_{2} \mathrm{O}(80 \%, 5.5 \mathrm{mmol})$, and the reaction mixture was stirred for 24 hours at room temperature. The reaction mixture was concentrated under reduced pressure, and the residue was purified by washing with cold ethanol $(3 \times 10 \mathrm{~mL})$, leading the pure derivative 6 in $78 \%$ yield.

Synthesis of tert-butyl (1S)-2-hydrazino-1-(hydroxymethyl)-2-oxoethylcarbamate 7

To a stirred solution of $5(0.3 \mathrm{~g}, 1.37 \mathrm{mmol})$ in ethanol $(10 \mathrm{~mL})$ at room temperature was added $\mathrm{N}_{2} \mathrm{H}_{4} \cdot \mathrm{H}_{2} \mathrm{O}(80 \%$, $5.5 \mathrm{mmol})$. The reaction mixture was stirred for 24 hours at room temperature and concentrated under reduced pressure. The residue was column chromatographed on silica gel using a gradient 0 to $5 \%$ metanol in chloroform, affording 7 as a white solid in $70 \%$ yield.

General procedure for the synthesis of benzyl (1S)-2-[2(substituted-benzylidene)hydrazino]-1-(hydroxymethyl)-2oxoethylcarbamate (8a-o) and tert-butyl (1S)-2-[2(substituted-benzylidene)hydrazino]-1-(hydroxymethyl)-2oxoethylcarbamate (9a-o)

To a stirred solution of 6 or $7(1.0 \mathrm{mmol})$ in ethanol (10 $\mathrm{mL}$ ) at room temperature was added the appropriate benzaldehyde $(1.05 \mathrm{mmol})$, and the reaction mixture was stirred for 4 hours at $80^{\circ} \mathrm{C}$. The reaction mixture was concentrated under reduced pressure, and the residue was purified by washing with cold ethanol $(3 \times 10 \mathrm{~mL})$, affording the derivatives 8a-o or 9a-o in $70-90 \%$ yield.

General procedure for the synthesis of (4S)- $N$ '[(substituted-phenyl)methylidene]-2-oxo-1,3-oxazolidine-4carbohydrazide 10a and $\mathbf{1 0 b}$.

To a stirred solution of $\mathbf{8 c}$ or $\mathbf{8 e}(1.0 \mathrm{mmol})$ in THF (10 $\mathrm{mL})$ at room temperature was added sodium hydride $(60 \%$ dispersion in mineral oil; $5 \mathrm{mmol}$ ), and the reaction mixture was stirred for 20 minutes at room temperature. The reaction mixture was concentrated, $\mathrm{CH}_{2} \mathrm{Cl}_{2}$ was added, washed with aqueous saturated $\mathrm{NH}_{4} \mathrm{Cl}$ solution, brine and dried over $\mathrm{Na}_{2} \mathrm{SO}_{4}$. The organic layer was concentrated and the residue was purified by column chromatography on silica gel using a gradient 0 to $1 \%$ methanol in dichloromethane, affording the derivatives 8a-o or 9a-o in $70-90 \%$ yield.

Analytical data for compounds $3,4,5,6,7,8 \mathbf{a}-\mathbf{0}, 9 \mathbf{a}-\mathbf{0}$ and 10a-b

Methyl (2S)-2-amino-3-hydroxypropanoate hydrochloride 3

Yield: 99\%. m.p. $163.1^{0} \mathrm{C} .{ }^{1} \mathrm{H}-\mathrm{NMR}(400 \mathrm{MHz}, \mathrm{DMSO}-$ $\left.d_{6}\right) \delta(\mathrm{ppm}): 8.60\left(3 \mathrm{H} ; \mathrm{s} ; \mathrm{NH}_{3}{ }^{+}\right) ; 5.64(1 \mathrm{H} ; \mathrm{s} ; \mathrm{OH}) ; 4.08(1 \mathrm{H}$; $\mathrm{t} ; J=3.6 ; \mathrm{CH}) ; 3.82\left(2 \mathrm{H} ; \mathrm{m} ; \mathrm{CH}_{2}\right) ; 3.73\left(3 \mathrm{H} ; \mathrm{s} ; \mathrm{CH}_{3}\right) .{ }^{13} \mathrm{C}-$ NMR $\left(100 \mathrm{MHz}, \mathrm{DMSO}-d_{6}\right) \delta(\mathrm{ppm}): 168.5 ; 59.5 ; 54.4 ; 52.8$. IR $\left(\mathrm{cm}^{-1} ; \mathrm{KBr}\right) v_{\max } 3362 ; 1743 . \mathrm{MS} / \mathrm{ESI}\left(\mathrm{m} / \mathrm{z}[\mathrm{M}+\mathrm{H}-\mathrm{HCl}]^{+}\right)$: 120.0

Methyl (2S)-2-[(benzyloxycarbonyl)amino]-3-hydroxypropanoate 4

Yield: 93\%. ${ }^{1} \mathrm{H}-\mathrm{NMR}\left(400 \mathrm{MHz}, \mathrm{CDCl}_{3}\right) \delta(\mathrm{ppm}): 7.36$ $(5 \mathrm{H} ; \mathrm{m} ; \mathrm{Ph}) ; 5.75(1 \mathrm{H} ; \mathrm{d} ; J=6.3 ; \mathrm{NH}) ; 5.12\left(2 \mathrm{H} ; \mathrm{s} ; \mathrm{CH}_{2} \mathrm{Ph}\right)$; $4.45(1 \mathrm{H} ; \mathrm{m} ; \mathrm{CH}) ; 4.00(1 \mathrm{H} ; \mathrm{dd} ; J=11.2$ and $J=3.2$; $\left.\mathrm{CH}^{\prime} \mathrm{OH}\right) ; 3.91(1 \mathrm{H} ; \mathrm{dd} ; J=11.2$ and $J=3.0 ; \mathrm{CHOH}) ; 3.78$ $\left(3 \mathrm{H} ; \mathrm{s} ; \mathrm{CH}_{3}\right) .{ }^{13} \mathrm{C}-\mathrm{NMR}\left(100 \mathrm{MHz}, \mathrm{CDCl}_{3}\right) \delta(\mathrm{ppm}): 171.8$; $157.0 ; 136.7 ; 129.2 ; 128.9 ; 128.7 ; 67.8 ; 63.7 ; 56.7 ; 53.3$. IR $\left(\mathrm{cm}^{-1}, \mathrm{KBr}\right) v_{\max } 3310 ; 1722 ; 1689 . \mathrm{MS} / \mathrm{ESI}\left(\mathrm{m} / \mathrm{z}[\mathrm{M}-\mathrm{H}]^{-}\right)$: 252.2

Methyl (2S)-2-[(tert-butoxycarbonyl)amino]-3-hydroxypropanoate 5

Yield: $85 \% .{ }^{1} \mathrm{H}-\mathrm{NMR}\left(500 \mathrm{MHz}, \mathrm{CDCl}_{3}\right) \delta(\mathrm{ppm}): 5.61$ $(1 \mathrm{H} ; \mathrm{d} ; J=6.8 ; \mathrm{NH}) ; 4.38(1 \mathrm{H} ; \mathrm{m} ; \mathrm{CH}) ; 3.96(1 \mathrm{H} ; \mathrm{dd} ; J=$ 11.2 and $\left.J=2.9, \mathrm{CH}^{\prime} \mathrm{OH}\right) ; 3.87(1 \mathrm{H} ; \mathrm{dd} ; J=11.2$ and $J=$ 3.4; $\mathrm{CHOH}) ; 3.78$ (3H; s; $\left.\mathrm{CH}_{3}\right) ; 1.45\left(9 \mathrm{H} ; \mathrm{s} ;\left(\mathrm{CH}_{3}\right)_{3} \mathrm{C}\right) .{ }^{13} \mathrm{C}-$ NMR $\left(125 \mathrm{MHz}, \mathrm{CDCl}_{3}\right) \delta(\mathrm{ppm}): 171.5 ; 155.8 ; 80.3 ; 63.3$; $55.7 ; 52.6 ; 28.3$. IR $\left(\mathrm{cm}^{-1} ; \mathrm{KBr}\right) v_{\max } 3300 ; 1746 ; 1694$. MS/ESI (m/z [M+Na $\left.]^{+}\right): 242.0$.

Benzyl (1S)-2-hydrazino-1-(hydroxymethyl)-2-oxoethylcarbamate 6

Yield: $78 \%$. m.p. $176^{0} \mathrm{C} .{ }^{1} \mathrm{H}-\mathrm{NMR}\left(500 \mathrm{MHz}, \mathrm{DMSO}-d_{6}\right)$ $\delta$ (ppm): $9.10\left(1 \mathrm{H} ; \mathrm{s} ; \mathrm{NHNH}_{2}\right) ; 7.32\left(5 \mathrm{H} ; \mathrm{m} ; \mathrm{PhCH}_{2}\right) ; 7.15$ $(1 \mathrm{H} ; \mathrm{d} ; J=8.2 ; \mathrm{N} \underline{\mathrm{HCH}}) ; 5.02\left(2 \mathrm{H} ; \mathrm{s} ; \mathrm{CH}_{2} \mathrm{Ph}\right) ; 4.85(1 \mathrm{H} ; \mathrm{t}$; $J=5.6 ; \mathrm{OH}) ; 4.20\left(2 \mathrm{H} ; \mathrm{s} ; \mathrm{NHNH}_{2}\right) ; 4.01(1 \mathrm{H} ; \mathrm{m} ; \mathrm{CH}) ; 3.60-$ $3.40\left(2 \mathrm{H} ; \mathrm{m} ; \mathrm{CH}_{2} \mathrm{OH}\right) .{ }^{13} \mathrm{C}-\mathrm{NMR}\left(125 \mathrm{MHz}, \mathrm{DMSO}-d_{6}\right) \delta$ (ppm): $169.4 ; 155.8 ; 137.0 ; 128.3 ; 127.8 ; 127.7 ; 65.5 ; 61.7$; 56.0. IR $\left(\mathrm{cm}^{-1} ; \mathrm{KBr}\right) v_{\max } 3286 ; 1692 ; 1653$. MS/ESI $(\mathrm{m} / \mathrm{z}$ $\left.[\mathrm{M}+\mathrm{Na}]^{+}\right): 276.0$

Tert-butyl (1S)-2-hydrazino-1-(hydroxymethyl)-2-oxoethylcarbamate 7

Yield: $70 \%$. m.p. $130^{0} \mathrm{C} .{ }^{1} \mathrm{H}-\mathrm{NMR}\left(500 \mathrm{MHz}, \mathrm{DMSO}-d_{6}\right)$ $\delta$ (ppm): $9.02\left(1 \mathrm{H} ; \mathrm{s} ; \mathrm{NHNH}_{2}\right) ; 6.58(1 \mathrm{H} ; \mathrm{d} ; J=8.2 ; \mathrm{N} \underline{\mathrm{HCH}})$; $4.81(1 \mathrm{H} ; \mathrm{t} ; J=5.6 ; \mathrm{OH}) ; 4.19\left(2 \mathrm{H} ; \mathrm{s} ; \mathrm{NHNH}_{2}\right) ; 3.93(1 \mathrm{H} ; \mathrm{m}$; $\mathrm{CH})$; 3.60-3.40 (2H; m; $\left.\mathrm{CH}_{2} \mathrm{OH}\right) ; 1.37\left(9 \mathrm{H} ; \mathrm{s} ;\left(\mathrm{CH}_{3}\right)_{3} \mathrm{C}\right)$. ${ }^{13} \mathrm{C}-\mathrm{NMR}\left(125 \mathrm{MHz}, \mathrm{DMSO}-d_{6}\right) \delta(\mathrm{ppm}): 169.7 ; 155.1 ; 78.1$; 61.9; 55.5; 28.2. IR ( $\left.\mathrm{cm}^{-1} ; \mathrm{KBr}\right) v_{\max } 3281 ; 1699 ; 1668$. MS/ESI (m/z [M-H] $): 218.1$ 
Benzyl(1S)-2-[(2E/Z)-2-benzylidenehydrazino]-1(hydroxymethyl)-2-oxoethylcarbamate 8a

Yield: $85 \%$. m.p. $157^{0} \mathrm{C} .{ }^{1} \mathrm{H}-\mathrm{NMR}\left(400 \mathrm{MHz}, \mathrm{DMSO}-d_{6}\right)$ $\delta(\mathrm{ppm}): 11.50$ and $11.42(1 \mathrm{H} ; \mathrm{s} ; \mathrm{NHN} ;(E / Z)$-isomer); 8.25 and $7.99(1 \mathrm{H} ; \mathrm{s} ; \mathrm{N}=\mathrm{CH} ;(E / Z)$-isomer); $7.68(2 \mathrm{H} ; \mathrm{m} ; \mathrm{H} 2$ and H6); 7.50-7.20 (9H; m; $\mathrm{N} \underline{\mathrm{HCH}}, \mathrm{PhCH}_{2}, \mathrm{H} 3, \mathrm{H} 4$ and $\left.\mathrm{H} 5\right)$; 5.05 and $5.04\left(2 \mathrm{H} ; \mathrm{s} ; \mathrm{CH}_{2} \mathrm{Ph}\right.$; (E/Z)-isomer); $5.03(\mathrm{~m})$ and $4.86(\mathrm{t} ; J=5.7),(1 \mathrm{H} ; \mathrm{OH} ;(E / Z)$-isomer $) ; 5.03$ and $4.15(1 \mathrm{H}$; $\mathrm{m}$; $\mathrm{CH}$; (E/Z)-isomer); 3.80-3.60 (2H; m; $\left.\mathrm{CH}_{2} \mathrm{OH}\right) .{ }^{13} \mathrm{C}-$ NMR $\left(100 \mathrm{MHz}\right.$, DMSO- $\left.d_{6}\right) \delta(\mathrm{ppm}): 171.3 ; 166.9 ; 155.9$; $147.0 ; 143.3 ; 137.0 ; 136.9 ; 134.2 ; 134.1 ; 130.0 ; 129.8$; $128.8,128.3 ; 127.8 ; 127.7 ; 127.0 ; 126.8 ; 65.5 ; 65.4 ; 61.5$; $61.1 ; 56.4$; 54.6. IR $\left(\mathrm{cm}^{-1} ; \mathrm{KBr}\right) v_{\max } 3387 ; 1678$. MS/ESI: [M-H]: 340.3

Benzyl (1S)-1-(hydroxymethyl)-2-[(2E/Z)-2-(2-nitrobenzylidene)hydrazino]-2-oxoethylcarbamate $\mathbf{8 b}$

Yield: $80 \%$. m.p. $155^{0} \mathrm{C} .{ }^{1} \mathrm{H}-\mathrm{NMR}\left(500 \mathrm{MHz}\right.$, DMSO- $\left.d_{6}\right)$ $\delta$ (ppm): 11.88 and $11.71(1 \mathrm{H} ; \mathrm{s} ; \mathrm{NHN}$; $(E / Z)$-isomer); 8.66 and $8.39(1 \mathrm{H} ; \mathrm{s} ; \mathrm{N}=\mathrm{CH} ;(E / Z)$-isomer $) ; 8.07(2 \mathrm{H} ; \mathrm{m} ; \mathrm{H} 3$ and H6); $7.80(1 \mathrm{H} ; \mathrm{m} ; \mathrm{H} 5) ; 7.67(1 \mathrm{H} ; \mathrm{m} ; \mathrm{H} 4) ; 7.44$ (d, $J=7.8)$ and $7.34(\mathrm{~m}),(1 \mathrm{H} ; \mathrm{NHCH} ;(E / Z)$-isomer $)$; 7.38-7.30 $(5 \mathrm{H} ; \mathrm{m}$; $\mathrm{Ph}) ; 5.05$ and $5.04\left(\overline{\mathrm{H}} ; \mathrm{s} ; \mathrm{C}_{2} \mathrm{Ph} ;(E / Z)\right.$-isomer); $5.03(\mathrm{~m})$ and $4.89(\mathrm{t} ; J=5.9),(1 \mathrm{H} ; \mathrm{OH} ;(E / Z)$-isomer $) ; 5.03$ and 4.15 $(1 \mathrm{H} ; \mathrm{m} ; \mathrm{CH} ;(E / Z)$-isomer $) ; 3.80-3.60\left(2 \mathrm{H} ; \mathrm{m} ; \mathrm{CH}_{2} \mathrm{OH}\right) .{ }^{13} \mathrm{C}-$ NMR $\left(125 \mathrm{MHz}\right.$, DMSO- $\left.d_{6}\right) \delta(\mathrm{ppm}): 171.7 ; 167.4 ; 156.0$; $148.2 ; 148.1 ; 142.4 ; 138.8 ; 137.0 ; 136.9 ; 133.8 ; 133.6$; $130.6 ; 130.5 ; 128.7 ; 128.4 ; 128.0 ; 127.8 ; 127.7 ; 124.7$; 124.6; 65.6; 65.4; 61.4; 61.1; 56.5; 54.4. IR $\left(\mathrm{cm}^{-1} ; \mathrm{KBr}\right) v_{\max }$ 3392; 1694; 1672; 1555; 1342. MS/ESI: [M-H]: 385.3

Benzyl (1S)-1-(hydroxymethyl)-2-[(2E/Z)-2-(3-nitrobenzylidene)hydrazino]-2-oxoethylcarbamate $8 \mathbf{c}$

Yield: $80 \%$. m.p. $175^{0} \mathrm{C} .{ }^{1} \mathrm{H}-\mathrm{NMR}\left(500 \mathrm{MHz}\right.$, DMSO- $\left.d_{6}\right)$ $\delta$ (ppm): 11.79 and $11.67(1 \mathrm{H} ; \mathrm{s} ; \mathrm{NHN}$; $(E / Z)$-isomer); 8.52 and $8.49(1 \mathrm{H} ; \mathrm{s} ; \mathrm{H} 2 ;(E / Z)$-isomer $) ; 8.37$ and $8.12(1 \mathrm{H}$; s; $\mathrm{N}=\mathrm{CH}$; $(E / Z)$-isomer $) ; 8.25(1 \mathrm{H} ; \mathrm{m} ; \mathrm{H} 4$ or $\mathrm{H} 6) ; 8.16(\mathrm{~d} ; \mathrm{J}=$ $7.4)$ and $8.13(\mathrm{~m}),(1 \mathrm{H}, \mathrm{H} 4$ or $\mathrm{H} 6$; $(E / Z)$-isomer $) ; .75(1 \mathrm{H}$; $\mathrm{m}$; H5); $7.45(\mathrm{~d} ; J=7.3)$ and $7.36(\mathrm{~m}),(1 \mathrm{H} ; \mathrm{NHCH} ;(E / Z)-$ isomer); 7.38-7.30 (5H; m; $\mathrm{Ph}) ; 5.05\left(2 \mathrm{H} ; \mathrm{s} ; \mathrm{CH}_{2} \mathrm{Ph}\right) ; 5.05$ $(\mathrm{m})$ and $4.91(\mathrm{t} ; J=5.9),(1 \mathrm{H} ; \mathrm{OH} ;(E / Z)$-isomer $) ; 5.05$ and 4.17 (1H; m; $\mathrm{CH}$; (E/Z)-isomer); 3.80-3.60 (2H; m; $\left.\mathrm{CH}_{2} \mathrm{OH}\right)$. ${ }^{13} \mathrm{C}-\mathrm{NMR}\left(125 \mathrm{MHz}\right.$, DMSO-d $\left.d_{6}\right) \delta(\mathrm{ppm}): 171.7 ; 167.4$; $155.9 ; 148.3 ; 148.2 ; 144.6 ; 141.2 ; 137.1 ; 136.9 ; 136.2$; $136.0 ; 133.3 ; 132.8 ; 130.4 ; 128.4 ; 128.3 ; 127.8 ; 127.7$; $124.2 ; 124.1 ; 121.2 ; 121.0 ; 65.6 ; 65.4 ; 61.5 ; 61.1 ; 56.4 ; 54.4$. IR $\left(\mathrm{cm}^{-1} ; \mathrm{KBr}\right) v_{\max } 3392 ; 1694 ; 1674 ; 1553 ; 1342$. MS/ESI: [M-H]: 385.3

Benzyl (1S)-1-(hydroxymethyl)-2-[(2E/Z)-2-(4-nitrobenzylidene)hydrazino]-2-oxoethylcarbamate 8d

Yield: $82 \%$. m.p. $190^{\circ} \mathrm{C} .{ }^{1} \mathrm{H}-\mathrm{NMR}\left(500 \mathrm{MHz}, \mathrm{DMSO}-d_{6}\right)$ $\delta(\mathrm{ppm})$ : 11.87 and $11.76(1 \mathrm{H}$; s; NHN; (E/Z)-isomer); 8.34$8.09(3 \mathrm{H} ; \mathrm{m} ; \mathrm{N}=\mathrm{CH}, \mathrm{H} 3$ and $\mathrm{H} 5) ; 7.98-7.95(2 \mathrm{H} ; \mathrm{m} ; \mathrm{H} 2$ and H6); $7.50(\mathrm{~d} ; J=7.7)$ and $7.36(\mathrm{~m}),(1 \mathrm{H} ; \mathrm{NHCH} ;(E / Z)-$ isomer); 7.40-7.30 (5H; m; Ph); $5.05\left(2 \mathrm{H} ; \mathrm{s} ; \mathrm{CH}_{2} \mathrm{Ph}\right) ; 5.04$ $(\mathrm{m})$ and $4.93(\mathrm{t} ; J=6.3),(1 \mathrm{H} ; \mathrm{OH} ;(E / Z)$-isomer); 5.04 and $4.16(1 \mathrm{H} ; \mathrm{m} ; \mathrm{CH} ;(E / Z)$-isomer $) ; 3.80-3.60\left(2 \mathrm{H} ; \mathrm{m} ; \mathrm{CH}_{2} \mathrm{OH}\right)$. ${ }^{13} \mathrm{C}-\mathrm{NMR}\left(125 \mathrm{MHz}, \mathrm{DMSO}-d_{6}\right) \delta(\mathrm{ppm}): 171.8 ; 167.4$; $155.9 ; 155.8 ; 147.7 ; 147.6 ; 144.4 ; 140.9 ; 140.5 ; 140.3$; $136.9 ; 136.8 ; 128.2 ; 127.9 ; 127.7 ; 127.6 ; 124.0 ; 65.5 ; 65.3$;
$61.3 ; 61.0 ; 56.3 ; 54.4$. IR $\left(\mathrm{cm}^{-1} ; \mathrm{KBr}\right) v_{\max } 1694 ; 1680 ; 1553$; 1348. MS/ESI: [M-H]: 385.2

Benzyl (1S)-2-[(2E/Z)-2-(3-cyanobenzylidene)hydrazino] -1-(hydroxymethyl)-2-oxoethylcarbamate $\mathbf{8 e}$

Yield: $82 \%$. m.p. $155^{0} \mathrm{C} .{ }^{1} \mathrm{H}-\mathrm{NMR}\left(400 \mathrm{MHz}\right.$, DMSO- $\left.d_{6}\right)$ $\delta(\mathrm{ppm}): 11.74$ and $11.60(1 \mathrm{H} ; \mathrm{s}$; NHN; $(E / Z)$-isomer); 8.30$8.00(3 \mathrm{H} ; \mathrm{m} ; \mathrm{N}=\mathrm{CH}, \mathrm{H} 2$ and $\mathrm{H} 6) ; 7.87(1 \mathrm{H} ; \mathrm{m} ; \mathrm{H} 4$ or $\mathrm{H} 5)$; $7.63(1 \mathrm{H} ; \mathrm{m} ; \mathrm{H} 4$ or $\mathrm{H} 5) ; 7.42(\mathrm{~d} ; J=7.8)$ and $7.40-7.25(\mathrm{~m})$, $(1 \mathrm{H} ; \mathrm{NHCH}$; $(E / Z)$-isomer); 7.40-7.25 $(5 \mathrm{H} ; \mathrm{m} ; \mathrm{Ph}) ; 5.04$ $\left(2 \mathrm{H} ; \mathrm{s} ; \mathrm{C}_{2} \mathrm{Ph}\right) ; 5.04(\mathrm{~m})$ and $4.90(\mathrm{t} ; J=6.1),(1 \mathrm{H} ; \mathrm{OH}$; $(E / Z)$-isomer $) ; 5.04$ and $4.15(1 \mathrm{H} ; \mathrm{m} ; \mathrm{CH} ;(E / Z)$-isomer $)$; 3.80-3.55 (2H; m; $\left.\mathrm{CH}_{2} \mathrm{OH}\right) .{ }^{13} \mathrm{C}-\mathrm{NMR}\left(100 \mathrm{MHz}, \mathrm{DMSO}-d_{6}\right)$ $\delta(\mathrm{ppm}): 171.7 ; 167.4 ; 156.0 ; 144.7 ; 141.1 ; 137.1 ; 136.9$; $135.6 ; 135.5 ; 133.2 ; 133.0 ; 131.3 ; 130.9 ; 130.8 ; 130.2$; $130.1 ; 128.4 ; 128.3 ; 127.9 ; 127.8 ; 127.7 ; 118.5 ; 118.4$; $112.0 ; 65.6 ; 65.4 ; 61.5 ; 61.1 ; 56.4 ; 54.5 . \mathrm{IR}\left(\mathrm{cm}^{-1} ; \mathrm{KBr}\right) v_{\max }$ 3291; 2232; 1676. MS/ESI: [M-H]: 365.3

Benzyl (1S)-2-[(2E/Z)-2-(4-cyanobenzylidene)hydrazino] -1-(hydroxymethyl)-2-oxoethylcarbamate $\mathbf{8 f}$

Yield: $80 \%$. m.p. $110^{0} \mathrm{C} .{ }^{1} \mathrm{H}-\mathrm{NMR}\left(500 \mathrm{MHz}\right.$, DMSO- $\left.d_{6}\right)$ $\delta$ (ppm): 11.81 and $11.70(1 \mathrm{H} ; \mathrm{s} ; \mathrm{NHN} ;(E / Z)$-isomer); 8.30 and $8.03(1 \mathrm{H} ; \mathrm{s} ; \mathrm{N}=\mathrm{CH} ;(E / Z)$-isomer $) ; 7.93-7.85(4 \mathrm{H} ; \mathrm{m}$; $\mathrm{H} 2, \mathrm{H} 3, \mathrm{H} 5$ and $\mathrm{H} 6) ; 7.48(\mathrm{~d}, J=7.8)$ and 7.40-7.30 (m), $(1 \mathrm{H}$; $\mathrm{NHCH}$; (E/Z)-isomer); 7.40-7.30 (5H; m; Ph); 5.04 $\left(2 \mathrm{H} ; \mathrm{s} ; \mathrm{CH}_{2} \mathrm{Ph}\right) ; 5.04(\mathrm{~m})$ and $4.92(\mathrm{dd} ; J=6.3$ and $J=5.4)$, $(1 \mathrm{H} ; \mathrm{OH} ;(E / Z)$-isomer); 5.04 and $4.15(1 \mathrm{H} ; \mathrm{m}$; $\mathrm{CH} ;(E / Z)$ isomer); $3.80-3.50\left(2 \mathrm{H} ; \mathrm{m} ; \mathrm{CH}_{2} \mathrm{OH}\right) .{ }^{13} \mathrm{C}-\mathrm{NMR}(125 \mathrm{MHz}$, DMSO- $\left.d_{6}\right) \delta$ (ppm): $171.8 ; 167.5 ; 156.0 ; 145.0 ; 141.4$; $138.8 ; 138.6 ; 137.1 ; 137.0 ; 132.8,128.4,128.2,127.9$, $127.8,127.6,127.5 ; 126.8 ; 118.8 ; 118.7 ; 111.9 ; 111.7 ; 65.6$; $65.4 ; 61.5 ; 61.1 ; 56.5 ; 54.6$. IR $\left(\mathrm{cm}^{-1} ; \mathrm{KBr}\right) v_{\max } 3293 ; 2229$; 1673. MS/ESI: [M-H]: 365.3

Benzyl (1S)-2-[(2E)-2-(2-hydroxybenzylidene)hydrazino]-1-(hydroxymethyl)-2-oxoethylcarbamate $\mathbf{8 g}$

Yield: $89 \%$. m.p.: $160^{\circ} \mathrm{C} .{ }^{1} \mathrm{H}-\mathrm{NMR}\left(500 \mathrm{MHz}\right.$, DMSO- $\left.d_{6}\right)$ $\delta(\mathrm{ppm}): 11.79(1 \mathrm{H} ; \mathrm{s} ; \mathrm{NHN}) ; 11.12(1 \mathrm{H} ; \mathrm{s} ; \mathrm{Ph}-\mathrm{OH}) ; 8.46$ $(1 \mathrm{H} ; \mathrm{s} ; \mathrm{N}=\mathrm{CH} ;(E)$-isomer); $7.52(1 \mathrm{H} ; \mathrm{dd} ; J=7.8$ and $J=1.5$; $\mathrm{H} 6) ; 7.48(1 \mathrm{H} ; \mathrm{d} ; J=7.8 ; \mathrm{NHCH}) ; 7.40-7.20(6 \mathrm{H} ; \mathrm{m} ; \mathrm{Ph}$ and $(\mathrm{H} 4$ or $\mathrm{H} 5)) ; 6.93-6.84(2 \mathrm{H} ; \mathrm{m} ; \mathrm{H} 3$ and $(\mathrm{H} 4$ or $\mathrm{H} 5)) ; 5.05$ $\left(3 \mathrm{H} ; \mathrm{m} ; \mathrm{CH}_{2} \mathrm{Ph}\right.$ and $\left.\mathrm{OH}\right) ; 4.13(1 \mathrm{H} ; \mathrm{m} ; \mathrm{CH}) ; 3.80-3.60(2 \mathrm{H}$; $\left.\mathrm{m} ; \mathrm{CH}_{2} \mathrm{OH}\right) .{ }^{13} \mathrm{C}-\mathrm{NMR}\left(125 \mathrm{MHz}\right.$, DMSO- $\left.d_{6}\right) \delta(\mathrm{ppm})$ : $171.6 ; 157.8 ; 156.4 ; 141.2 ; 137.4 ; 131.9,131.6,129.8$, $128.8,128.3 ; 128.2 ; 126.7 ; 119.9 ; 119.1 ; 116.6 ; 65.9 ; 61.5$; 55.0. IR ( $\left.\mathrm{cm}^{-1} ; \mathrm{KBr}\right) v_{\max } 3312 ; 1681$. MS/ESI: [M-H]: 356.3

Benzyl (1S)-2-[(2E)-2-(2,3-dihydroxybenzylidene)hydrazino]-1-(hydroxymethyl)-2-oxoethylcarbamate $\mathbf{8 h}$

Yield: $81 \%$. m.p. $150^{0} \mathrm{C} .{ }^{1} \mathrm{H}-\mathrm{NMR}\left(500 \mathrm{MHz}\right.$, DMSO- $\left.d_{6}\right)$ $\delta(\mathrm{ppm}): 11.76(1 \mathrm{H} ; \mathrm{s} ; \mathrm{NHN}) ; 10.95(1 \mathrm{H} ; \mathrm{s} ; \mathrm{C} 1-\mathrm{OH}$ or $\mathrm{C} 2-\mathrm{OH}) ; 9.21(1 \mathrm{H}$; s; $\mathrm{C} 1-\mathrm{OH}$ or $\mathrm{C} 2-\mathrm{OH}) ; 8.41(1 \mathrm{H}$; s; $\mathrm{N}=\mathrm{CH} ;(E)$-isomer); $7.44(1 \mathrm{H} ; \mathrm{d} ; J=7.4 ; \mathrm{NHCH}) ; 7.40-7.20$ $(5 \mathrm{H} ; \mathrm{m} ; \mathrm{Ph}) ; 6.94(1 \mathrm{H} ; \mathrm{d} ; J=7.8 ; \mathrm{H} 6) ; 6.85-6.80(1 \mathrm{H} ; \mathrm{m}$; $\mathrm{H} 4) ; 6.73(1 \mathrm{H} ; \mathrm{t} ; J=7.8 ; \mathrm{H} 5) ; 5.04\left(3 \mathrm{H} ; \mathrm{m} ; \mathrm{CH}_{2} \mathrm{Ph}\right.$ and $\left.\mathrm{OH}\right)$; $4.15(1 \mathrm{H} ; \mathrm{m} ; \mathrm{CH}) ; 3.75-3.55\left(2 \mathrm{H} ; \mathrm{m} ; \mathrm{CH}_{2} \mathrm{OH}\right) .{ }^{13} \mathrm{C}-\mathrm{NMR}$ $\left(125 \mathrm{MHz}, \mathrm{DMSO}-d_{6}\right) \delta(\mathrm{ppm}): 171.1 ; 156.0 ; 145.6 ; 145.2$; $141.6 ; 136.9 ; 128.4 ; 127.9 ; 127.8 ; 127.7 ; 120.0 ; 119.2$; $117.4 ; 116.5 ; 65.4 ; 61.4 ; 56.3$. IR $\left(\mathrm{cm}^{-1} ; \mathrm{KBr}\right) v_{\max } 3270$; 1676. MS/ESI: [M-H]: 372.3 
Benzyl (1S)-2-[(2E)-2-(2,4-dihydroxybenzylidene)hydrazino]-1-(hydroxymethyl)-2-oxoethylcarbamate $\mathbf{8 i}$

Yield: $85 \%$. m.p. $166^{\circ} \mathrm{C} .{ }^{1} \mathrm{H}-\mathrm{NMR}\left(500 \mathrm{MHz}, \mathrm{DMSO}-d_{6}\right)$ $\delta(\mathrm{ppm}): 11.60(1 \mathrm{H} ; \mathrm{s} ; \mathrm{NHN}) ; 11.30(1 \mathrm{H} ; \mathrm{s} ; \mathrm{C} 1-\mathrm{OH}$ or $\mathrm{C} 3-\mathrm{OH}) ; 10.00(1 \mathrm{H} ; \mathrm{s} ; \mathrm{C} 1-\mathrm{OH}$ or $\mathrm{C} 3-\mathrm{OH}) ; 8.31(1 \mathrm{H} ; \mathrm{s}$; $\mathrm{N}=\mathrm{CH} ;(E)$-isomer); $7.45(1 \mathrm{H} ; \mathrm{d} ; J=7.8 ; \mathrm{H} 6) ; 7.40-7.30(5 \mathrm{H}$; $\mathrm{m} ; \mathrm{Ph}) ; 7.29(1 \mathrm{H} ; \mathrm{d} ; J=8.5 ; \mathrm{NHCH}) ; 6.37-6.27(2 \mathrm{H} ; \mathrm{m} ; \mathrm{H} 3$ and $\mathrm{H} 5) ; 5.05\left(3 \mathrm{H} ; \mathrm{m} ; \mathrm{CH}_{2} \mathrm{Ph}\right.$ and $\left.\mathrm{OH}\right) ; 4.10(1 \mathrm{H} ; \mathrm{m} ; \mathrm{CH})$; 3.75-3.55 (2H; m; $\left.\mathrm{CH}_{2} \mathrm{OH}\right) .{ }^{13} \mathrm{C}-\mathrm{NMR}\left(125 \mathrm{MHz}\right.$, DMSO- $\left.d_{6}\right)$ $\delta(\mathrm{ppm}): 171.1 ; 161.1 ; 158.4 ; 156.4 ; 142.2 ; 137.4 ; 131.7$, 128.8, 128.4, 128.3; 128.2; 128.1; 110.9; 108.1; 103.0; 65.8; 61.4; 54.9. IR $\left(\mathrm{cm}^{-1} ; \mathrm{KBr}\right) v_{\max } 3412 ; 3312 ; 1677 . \mathrm{MS} / \mathrm{ESI}:$ [M-H]: 372.3

Benzyl (1S)-2-[(2E)-2-(2,5-dihydroxybenzylidene)hydrazino]-1-(hydroxymethyl)-2-oxoethylcarbamate $\mathbf{8 j}$

Yield: $87 \%$. m.p. $145^{0} \mathrm{C} .{ }^{1} \mathrm{H}-\mathrm{NMR}\left(500 \mathrm{MHz}, \mathrm{DMSO}-d_{6}\right)$ $\delta(\mathrm{ppm}): 11.64(1 \mathrm{H} ; \mathrm{s} ; \mathrm{NHN}) ; 10.23(1 \mathrm{H} ; \mathrm{s} ; \mathrm{C} 1-\mathrm{OH}$ or $\mathrm{C} 4-\mathrm{OH}) ; 8.97(1 \mathrm{H} ; \mathrm{s} ; \mathrm{C} 1-\mathrm{OH}$ or $\mathrm{C} 4-\mathrm{OH}) ; 8.37(1 \mathrm{H} ; \mathrm{s}$; $\mathrm{N}=\mathrm{CH} ;(E)$-isomer); 7.40-7.30 $(5 \mathrm{H} ; \mathrm{m} ; \mathrm{Ph}) ; 7.40(1 \mathrm{H} ; \mathrm{d} ; \mathrm{J}=$ 8.3; $\mathrm{NHCH}) ; 6.93(1 \mathrm{H} ; \mathrm{d} ; J=2.0 ; \mathrm{H} 6) ; 6.75-6.65(2 \mathrm{H} ; \mathrm{m} ; \mathrm{H} 3$ and $\mathrm{H} 4) ; 5.05\left(2 \mathrm{H} ; \mathrm{s} ; \mathrm{CH}_{2} \mathrm{Ph}\right) ; 5.10-5.00(2 \mathrm{H} ; \mathrm{m} ; \mathrm{OH}$ and $\mathrm{CH})$; $3.75-3.55\left(2 \mathrm{H} ; \mathrm{m} ; \mathrm{C}_{2} \mathrm{OH}\right) .{ }^{13} \mathrm{C}-\mathrm{NMR}(125 \mathrm{MHz}$, DMSO- $\left.d_{6}\right) \delta$ (ppm): $171.0 ; 155.9 ; 150.0 ; 149.4 ; 141.0$; $136.9 ; 128.4 ; 127.9 ; 127.8 ; 127.7 ; 120.4 ; 118.9 ; 117.0$; $113.7 ; 111.1 ; 65.4 ; 61.1 ; 54.4$. IR $\left(\mathrm{cm}^{-1} ; \mathrm{KBr}\right) v_{\max } 3327$; 1664. MS/ESI: [M-H]: 372.3

Benzyl(1S)-2-[(2E)-2-(2-hydroxy-3-methoxybenzylidene)hydrazino]-1-(hydroxymethyl)-2-oxoethylcarbamate 8k

Yield: $80 \%$. m.p. $140^{\circ} \mathrm{C} .{ }^{1} \mathrm{H}-\mathrm{NMR}\left(500 \mathrm{MHz}, \mathrm{DMSO}-d_{6}\right)$ $\delta(\mathrm{ppm}): 11.72(1 \mathrm{H} ; \mathrm{s} ; \mathrm{NHN}) ; 10.80(1 \mathrm{H} ; \mathrm{s} ; \mathrm{C} 1-\mathrm{OH}) ; 8.46$ $(1 \mathrm{H} ; \mathrm{s} ; \mathrm{N}=\mathrm{CH} ;(E)$-isomer); $7.42(1 \mathrm{H} ; \mathrm{d}, J=7.8 ; \mathrm{N} \underline{\mathrm{HCH}})$; 7.40-7.30 $(5 \mathrm{H} ; \mathrm{m} ; \mathrm{Ph}) ; 7.10(1 \mathrm{H} ; \mathrm{dd} ; J=8.0$ and $J=1.1 ; \mathrm{H} 6)$; $7.02(1 \mathrm{H} ; \mathrm{d} ; J=8.0 ; \mathrm{H} 4) ; 6.85(1 \mathrm{H} ; \mathrm{t} ; J=8.0 ; \mathrm{H} 5) ; 5.04(2 \mathrm{H}$; s; $\left.\mathrm{CH}_{2} \mathrm{Ph}\right) ; 5.10-4.95(1 \mathrm{H} ; \mathrm{m} ; \mathrm{OH}) ; 4.13(1 \mathrm{H} ; \mathrm{m} ; \mathrm{CH}) ; 3.80$ $\left(3 \mathrm{H} ; \mathrm{s} ; \mathrm{CH}_{3}\right) ; 3.70-3.60\left(2 \mathrm{H} ; \mathrm{m} ; \mathrm{CH}_{2} \mathrm{OH}\right) .{ }^{13} \mathrm{C}-\mathrm{NMR}$ $\left(125 \mathrm{MHz}, \mathrm{DMSO}-d_{6}\right) \delta(\mathrm{ppm}): 171.1 ; 156.0 ; 147.9 ; 145.9$; $140.7 ; 137.0 ; 128.4 ; 127.8 ; 127.7 ; 127.6 ; 120.7 ; 119.2$; $118.8 ; 113.8 ; 65.4 ; 61.0 ; 55.9 ; 54.5 . \mathrm{IR}\left(\mathrm{cm}^{-1} ; \mathrm{KBr}\right) v_{\max }$ 3350; 1681. MS/ESI: [M-H]: 386.4

Benzyl (1S)-2-[(2E)-2-(3,4-dihydroxybenzylidene)hydrazino]-1-(hydroxymethyl)-2-oxoethylcarbamate $\mathbf{8 1}$

Yield: $80 \%$. m.p. $105^{0} \mathrm{C} .{ }^{1} \mathrm{H}-\mathrm{NMR}\left(500 \mathrm{MHz}, \mathrm{DMSO}-d_{6}\right)$ $\delta(\mathrm{ppm}): 11.24(1 \mathrm{H} ; \mathrm{s} ; \mathrm{NHN}) ; 9.30(2 \mathrm{H} ; \mathrm{s} ; \mathrm{C} 2-\mathrm{OH}$ and $\mathrm{C} 3-\mathrm{OH}) ; 8.04(1 \mathrm{H} ; \mathrm{s} ; \mathrm{N}=\mathrm{CH}$; $(E)$-isomer $) ; 7.40-7.30(6 \mathrm{H}$; $\mathrm{m} ; \mathrm{Ph}$ and $\mathrm{NHCH}) ; 7.18(1 \mathrm{H} ; \mathrm{d} ; J=1.6 ; \mathrm{H} 2) ; 6.89(1 \mathrm{H} ; \mathrm{m}$; H6); $6.78\left(1 \mathrm{H} ; \mathrm{s}\right.$; H5); $5.04\left(2 \mathrm{H}\right.$; s; $\left.\mathrm{C}_{2} \mathrm{Ph}\right) ; 5.10-4.95(1 \mathrm{H}$; $\mathrm{m} ; \mathrm{OH}) ; 4.10(1 \mathrm{H} ; \mathrm{m} ; \mathrm{CH}) ; 3.75-3.55\left(2 \mathrm{H} ; \mathrm{m} ; \mathrm{CH}_{2} \mathrm{OH}\right)$. ${ }^{13} \mathrm{C}-\mathrm{NMR}\left(125 \mathrm{MHz}\right.$ ，DMSO- $\left.d_{6}\right) \delta$ (ppm): 171.3; 156.4; $148.4 ; 147.9,146.2 ; 137.4 ; 128.8 ; 128.3 ; 128.2 ; 127.5$; $126.1 ; 126.0 ; 121.0 ; 116.0 ; 113.1 ; 66.0 ; 62.1 ; 56.8$. IR $\left(\mathrm{cm}^{-1}\right.$; KBr) $v_{\max } 3382$; 1686. MS/ESI: [M-H]: 372.3

Benzyl (1S)-2-[(2E)-2-(3,4-dimethoxybenzylidene)hydrazino]-1-(hydroxymethyl)-2-oxoethylcarbamate 8m

Yield: $83 \%$. m.p. $150^{0} \mathrm{C} .{ }^{1} \mathrm{H}-\mathrm{NMR}\left(500 \mathrm{MHz}, \mathrm{DMSO}-d_{6}\right)$ $\delta$ (ppm): $11.38(1 \mathrm{H} ; \mathrm{s} ; \mathrm{NHN}) ; 8.16(1 \mathrm{H} ; \mathrm{s} ; \mathrm{N}=\mathrm{CH} ;(E)-$ isomer); 7.40-7.25 (7H; m; $\mathrm{Ph}, \mathrm{NHCH}$ and $\mathrm{H} 2) ; 7.17(1 \mathrm{H}$; m; H6); $7.01(1 \mathrm{H} ; \mathrm{m} ; \mathrm{H} 5) ; 5.04\left(2 \mathrm{H} ; \mathrm{s} ; \mathrm{C}_{2} \mathrm{Ph}\right) ; 5.05-4.95$
$(2 \mathrm{H} ; \mathrm{m} ; \mathrm{OH}$ and $\mathrm{CH}) ; 3.80\left(6 \mathrm{H} ; \mathrm{s}, \mathrm{C} 2-\mathrm{OCH}_{3}\right.$ and $\left.\mathrm{C} 3-\mathrm{OCH}_{3}\right) ; \quad 3.80-3.55 \quad\left(2 \mathrm{H} ; \quad \mathrm{m} ; \quad \mathrm{C}_{2} \mathrm{OH}\right) .{ }^{13} \mathrm{C}-\mathrm{NMR}$ $\left(125 \mathrm{MHz}, \mathrm{DMSO}-d_{6}\right) \delta(\mathrm{ppm}): 171.1 ; 155.9 ; 150.5 ; 149.0$; $143.3 ; 137.1 ; 128.4,128.3,127.8,127.7 ; 127.0 ; 126.9$; $121.0 ; 111.5 ; 108.7 ; 65.4 ; 61.0 ; 55.6 ; 54.5 . \mathrm{IR}\left(\mathrm{cm}^{-1} ; \mathrm{KBr}\right)$ $v_{\max } 3291 ; 3203 ; 1728 ; 1666$. MS/ESI: [M-H]: 400.3

Benzyl (1S)-2-[(2E)-2-(2-hydroxy-4-methoxybenzylidene)hydrazino]-1-(hydroxymethyl)-2-oxoethylcarbamate 8n

Yield: $80 \%$. m.p. $162^{0} \mathrm{C} .{ }^{1} \mathrm{H}-\mathrm{NMR}\left(500 \mathrm{MHz}\right.$, DMSO- $d_{6}$ ) $\delta(\mathrm{ppm}): 11.68(1 \mathrm{H} ; \mathrm{s} ; \mathrm{NHN}) ; 11.45(1 \mathrm{H} ; \mathrm{s} ; \mathrm{C} 1-\mathrm{OH}) ; 8.36$ $(1 \mathrm{H} ; \mathrm{s} ; \mathrm{N}=\mathrm{CH} ;(E)$-isomer $) ; 7.47(1 \mathrm{H} ; \mathrm{d} ; J=7.8 ; \mathrm{H} 6) ; 7.40$ $(1 \mathrm{H} ; \mathrm{d} ; J=8.4 ; \mathrm{NHCH}) ; 7.38-7.25(5 \mathrm{H} ; \mathrm{m} ; \mathrm{Ph}) ; 6.55-6.40$ $(2 \mathrm{H} ; \mathrm{m} ; \mathrm{H} 3$ and $\mathrm{H} 5) ; 5.04\left(2 \mathrm{H} ; \mathrm{s} ; \mathrm{CH}_{2} \mathrm{Ph}\right) ; 5.10-5.00(1 \mathrm{H} ; \mathrm{m}$; $\mathrm{OH}) ; 4.11(1 \mathrm{H} ; \mathrm{m} ; \mathrm{CH}) ; 3.76\left(3 \mathrm{H} ; \mathrm{s} ; \mathrm{CH}_{3}\right) ; 3.70-3.55(2 \mathrm{H}$; $\left.\mathrm{m} ; \mathrm{CH}_{2} \mathrm{OH}\right) .{ }^{13} \mathrm{C}-\mathrm{NMR}\left(125 \mathrm{MHz}\right.$, DMSO- $\left.d_{6}\right) \delta(\mathrm{ppm})$ : $171.2 ; 162.5 ; 158.3 ; 156.4 ; 141.7 ; 137.4 ; 131.5,128.8$, 128.7, 128.3, 128.2; 127.6; 112.1; 106.9; 101.6; 66.0; 61.9; 55.8; 54.9. IR $\left(\mathrm{cm}^{-1} ; \mathrm{KBr}\right) v_{\max } 3329 ; 1678$. MS/ESI: [M-H]: 386.3

Benzyl (1S)-1-(hydroxymethyl)-2-[(2E)-2-(2-methoxybenzylidene)hydrazino]-2-oxoethylcarbamate 80

Yield: $80 \%$. m.p. $180^{\circ} \mathrm{C} .{ }^{1} \mathrm{H}-\mathrm{NMR}\left(400 \mathrm{MHz}\right.$, DMSO- $\left.d_{6}\right)$ $\delta(\mathrm{ppm}): 11.48(1 \mathrm{H} ; \mathrm{s} ; \mathrm{NHN}) ; 8.59(1 \mathrm{H} ; \mathrm{s} ; \mathrm{N}=\mathrm{CH} ;(E)-$ isomer); $7.83(1 \mathrm{H}$; $\mathrm{d}$; $\mathrm{J}=7.1$; $\mathrm{H} 5) ; 7.45-7.25(6 \mathrm{H} ; \mathrm{m}$; Ph e $\mathrm{H} 3)$; 7.45-7.25 (1H; m; NHCH); $7.10(1 \mathrm{H} ; \mathrm{s} ; \mathrm{H} 2) ; 7.00(1 \mathrm{H}$; $\mathrm{m} ; \mathrm{H} 4) ; 5.05\left(2 \mathrm{H} ; \mathrm{s} ; \mathrm{CH}_{2} \mathrm{Ph}\right) ; 5.01(1 \mathrm{H} ; \mathrm{m} ; \mathrm{CH}) ; 4.97(1 \mathrm{H} ; \mathrm{t}$; $J=5.8 ; \mathrm{OH}) ; 3.86\left(3 \mathrm{H} ; \mathrm{s} ; \mathrm{CH}_{3}\right) ; 3.80-3.55\left(2 \mathrm{H} ; \mathrm{m} ; \mathrm{CH}_{2} \mathrm{OH}\right)$. ${ }^{13} \mathrm{C}-\mathrm{NMR}\left(100 \mathrm{MHz}, \mathrm{DMSO}-d_{6}\right) \delta(\mathrm{ppm}): 171.2 ; 157.7$; $155.9 ; 142.5 ; 137.0 ; 131.5 ; 131.3 ; 128.3 ; 127.7 ; 127.6 ; 125.4$; $122.1 ; 120.7 ; 111.8 ; 65.5 ; 61.5 ; 56.4 ; 55.7$. IR $(\mathrm{cm}-1 ; \mathrm{KBr})$ $v_{\max } 3262 ; 1692$. MS/ESI: [M+Na]: 370.2.

Tert-butyl (1S)-2-[(2E/Z)-2-benzylidenehydrazino]-1(hydroxymethyl)-2- oxoethylcarbamate 9a

Yield: $82 \%$. m.p. $165^{0} \mathrm{C} .{ }^{1} \mathrm{H}-\mathrm{NMR}\left(400 \mathrm{MHz}\right.$, DMSO- $\left.d_{6}\right)$ $\delta$ (ppm): 11.44 and $11.35(1 \mathrm{H} ; \mathrm{s} ; \mathrm{NHN} ;(E / Z)$-isomer $) ; 8.24$ and $7.98(1 \mathrm{H} ; \mathrm{s} ; \mathrm{N}=\mathrm{CH} ;(E / Z)$-isomer $) ; 7.68(2 \mathrm{H} ; \mathrm{m} ; \mathrm{H} 2$ and $\mathrm{H} 6) ; 7.42(3 \mathrm{H} ; \mathrm{m} ; \mathrm{H} 3, \mathrm{H} 4$ and $\mathrm{H} 5) ; 6.78(\mathrm{~d} ; J=7.5)$ and 6.63 $(\mathrm{d} ; J=8.2),(1 \mathrm{H} ; \mathrm{NHCH} ;(E / Z)$-isomer $) ; 4.93(\mathrm{~m})$ and 4.80 $(\mathrm{t} ; J=5.8),(1 \mathrm{H} ; \mathrm{OH} ;(E / Z)$-isomer $) ; 4.93$ and $4.05(1 \mathrm{H} ; \mathrm{m}$; $\mathrm{CH}$; (E/Z)-isomer); 3.80-3.55 (2H; m; $\left.\mathrm{CH}_{2} \mathrm{OH}\right) ; 1,38$ (9H; s; $\left.\left(\mathrm{CH}_{3}\right)_{3} \mathrm{C}\right) .{ }^{13} \mathrm{C}-\mathrm{NMR}\left(100 \mathrm{MHz}, \mathrm{DMSO}-d_{6}\right) \delta(\mathrm{ppm}): 171.6$; $167.2 ; 155.2 ; 146.7 ; 143.1 ; 134.2 ; 134.1 ; 130.0 ; 129.8$; $128.8 ; 127.0 ; 126.8 ; 78.2 ; 78.0 ; 61.6 ; 61.2 ; 56.0 ; 54.1 ; 28.2$.

IR $\left(\mathrm{cm}^{-1} ; \mathrm{KBr}\right) v_{\max } 3370 ; 1684$. MS/ESI: [M-H]: 306.3

Tert-butyl (1S)-1-(hydroxymethyl)-2-[(2E/Z)-2-(2-nitrobenzylidene)hydrazino]-2-oxoethylcarbamate $9 \mathbf{b}$

Yield: $85 \%$. m.p. $155^{0} \mathrm{C} .{ }^{1} \mathrm{H}-\mathrm{NMR}\left(500 \mathrm{MHz}, \mathrm{DMSO}-d_{6}\right)$ $\delta$ (ppm): 11.82 and 11.65 (1H; s; NHN; (E/Z)-isomer); 8.65 and $8.38(1 \mathrm{H} ; \mathrm{s} ; \mathrm{N}=\mathrm{CH} ;(E / Z)$-isomer $) ; 8.06(2 \mathrm{H} ; \mathrm{m} ; \mathrm{H} 3$ and H6); $7.80(1 \mathrm{H} ; \mathrm{m} ; \mathrm{H} 5) ; 7.66(1 \mathrm{H} ; \mathrm{m} ; \mathrm{H} 4) ; 6.85$ (d; $J=7.6)$ and $6.70(\mathrm{~d} ; J=8.4),(1 \mathrm{H} ; \mathrm{N} \underline{\mathrm{HCH}} ;(E / Z)$-isomer); $4.97(\mathrm{t} ; J=$ $5.5)$ and $4.82(\mathrm{t} ; J=6.0),(1 \mathrm{H} ; \mathrm{OH} ;(E / Z)$-isomer $) ; 4.91$ and $4.04\left(1 \mathrm{H} ; \mathrm{m} ; \mathrm{CH} ;(E / Z)\right.$-isomer); 3.75-3.55 $\left(2 \mathrm{H} ; \mathrm{m} ; \mathrm{CH}_{2} \mathrm{OH}\right)$; 1.39 and $1.38\left(9 \mathrm{H} ; \mathrm{s} ;\left(\mathrm{CH}_{3}\right)_{3} \mathrm{C} ;(E / \mathrm{Z})\right.$-isomer $) .{ }^{13} \mathrm{C}-\mathrm{NMR}$ $\left(125 \mathrm{MHz}, \mathrm{DMSO}-d_{6}\right) \delta(\mathrm{ppm}): 172.0 ; 167.7 ; 155.3 ; 155.2$; $148.2 ; 148.0 ; 142.1 ; 138.6 ; 133.7 ; 133.6 ; 130.6 ; 130.4$; $128.7 ; 128,0 ; 127.9 ; 124.7 ; 124.6 ; 78.3 ; 78.1 ; 61.5 ; 61.2$; 
56.1; 54.0; 28.2. IR $\left(\mathrm{cm}^{-1} ; \mathrm{KBr}\right) v_{\max } 3238 ; 1705 ; 1524$; 1363. MS/ESI: [M-H] : 351.3

Tert-butyl (1S)-1-(hydroxymethyl)-2-[(2E/Z)-2-(3nitrobenzylidene)hydrazino]-2-oxoethylcarbamate 9c

Yield: $78 \%$. m.p. $172^{0} \mathrm{C} .{ }^{1} \mathrm{H}-\mathrm{NMR}\left(500 \mathrm{MHz}, \mathrm{DMSO}-d_{6}\right)$ $\delta$ (ppm): 11.72 and $11.59(1 \mathrm{H} ; \mathrm{s} ; \mathrm{NHN}$; (E/Z)-isomer); 8.52 and $8.47(1 \mathrm{H} ; \mathrm{s} ; \mathrm{H} 2 ;(E / Z)$-isomer $) ; 8.36$ and $8.11(1 \mathrm{H}$; s; $\mathrm{N}=\mathrm{CH} ;(E / Z)$-isomer $) ; 8.24(1 \mathrm{H} ; \mathrm{m} ; \mathrm{H} 4$ or $\mathrm{H} 6) ; 8.16(\mathrm{~d} ; J=$ $7.8)$ and $8.12(\mathrm{~m}),(1 \mathrm{H} ; \mathrm{H} 4$ or $\mathrm{H} 6 ;(E / Z)$-isomer); $7.73(1 \mathrm{H}$; $\mathrm{m} ; \mathrm{H} 5) ; 6.85(\mathrm{~d} ; J=7.4)$ and $6.72(\mathrm{~d} ; J=8.8),(1 \mathrm{H} ; \mathrm{N} \underline{\mathrm{HCH}}$; $(E / Z)$-isomer $) ; 4.97(\mathrm{~m})$ and $4.83(\mathrm{t} ; J=5.9),(1 \mathrm{H} ; \mathrm{m} ; \mathrm{OH}$; $(E / Z)$-isomer); 4.97 and $4.07(1 \mathrm{H} ; \mathrm{m}$; $\mathrm{CH}$; $(E / Z)$-isomer); 3.75-3.55 (2H; m; $\left.\mathrm{CH}_{2} \mathrm{OH}\right) ; 1.39$ and $1.38\left(9 \mathrm{H} ; \mathrm{s} ;\left(\mathrm{CH}_{3}\right)_{3} \mathrm{C}\right.$; (E/Z)-isomer). ${ }^{13} \mathrm{C}-\mathrm{NMR}\left(125 \mathrm{MHz}, \mathrm{DMSO}-d_{6}\right) \delta(\mathrm{ppm})$ : $172.0 ; 167.7 ; 155.3 ; 155.2 ; 148.3 ; 148.2 ; 144.3 ; 141.0$; $136.2 ; 136.0 ; 133.2 ; 132.8 ; 130.4,124.2,124.1 ; 121.1$; $121.0 ; 78.3 ; 78.1 ; 61.6 ; 61.2 ; 56.0 ; 54.0 ; 28.2$. IR $\left(\mathrm{cm}^{-1}\right.$; $\mathrm{KBr}) v_{\max } 3374 ; 1682 ; 1558 ; 1350$. MS/ESI: [M-H]: 351.2

Tert-butyl (1S)-1-(hydroxymethyl)-2-[(2E/Z)-2-(4nitrobenzylidene)hydrazino]-2-oxoethylcarbamate 9d

Yield: $80 \%$. m.p. $150^{0} \mathrm{C} .{ }^{1} \mathrm{H}-\mathrm{NMR}\left(500 \mathrm{MHz}, \mathrm{DMSO}-d_{6}\right)$ $\delta$ (ppm): 11.76 and $11.65(1 \mathrm{H} ; \mathrm{s} ; \mathrm{NHN}$; (E/Z)-isomer); 8.35 and $8.09(1 \mathrm{H} ; \mathrm{s} ; \mathrm{N}=\mathrm{CH} ;(E / Z)$-isomer $) ; 8.27(2 \mathrm{H} ; \mathrm{m} ; \mathrm{H} 3$ and $\mathrm{H} 5) ; 7.95(2 \mathrm{H} ; \mathrm{m} ; \mathrm{H} 2$ and $\mathrm{H} 6) ; 6.84(\mathrm{~d} ; J=7.3)$ and $6.72(\mathrm{~d}$; $J=8.0),(1 \mathrm{H} ; \mathrm{NHCH} ;(E / Z)$-isomer $) ; 4.98(\mathrm{~m})$ and $4.83(\mathrm{t} ; J=$ 6.0), $(1 \mathrm{H} ; \mathrm{OH} ;(E / Z)$-isomer $) ; 4.98$ and $4.07(1 \mathrm{H} ; \mathrm{m} ; \mathrm{CH}$; $(E / Z)$-isomer); 3.75-3.55 (2H; m; $\left.\mathrm{CH}_{2} \mathrm{OH}\right), 1.38(9 \mathrm{H} ; \mathrm{s}$; $\left.\left(\mathrm{CH}_{3}\right)_{3} \mathrm{C}\right) .{ }^{13} \mathrm{C}-\mathrm{NMR}\left(125 \mathrm{MHz}, \mathrm{DMSO}-d_{6}\right) \delta(\mathrm{ppm}): 172.0$; $167.7 ; 155.2 ; 147.8 ; 147.7 ; 144.3 ; 140.8 ; 140.6 ; 140.5$; $127.9 ; 127.7 ; 124.0 ; 78.3 ; 78.1 ; 61.5 ; 61.2 ; 56.0 ; 54.0 ; 28.1$. IR $\left(\mathrm{cm}^{-1} ; \mathrm{KBr}\right) v_{\max } 3370 ; 1684 ; 1562 ; 1344$. MS/ESI: [M$\mathrm{H}]: 351.3$

Tert-butyl (1S)-2-[(2E/Z)-2-(3-cyanobenzylidene)hydrazino]-1-(hydroxymethyl)-2-oxoethylcarbamate $9 e$

Yield: $78 \%$. m.p. $167^{0} \mathrm{C} .{ }^{1} \mathrm{H}-\mathrm{NMR}\left(400 \mathrm{MHz}, \mathrm{DMSO}-d_{6}\right)$ $\delta$ (ppm): 11.67 and $11.53(1 \mathrm{H}$; s; NHN; (E/Z)-isomer); 8.30$8.00(3 \mathrm{H} ; \mathrm{m} ; \mathrm{N}=\mathrm{CH}, \mathrm{H} 2$ and $\mathrm{H} 6) ; 7.86(1 \mathrm{H} ; \mathrm{m} ; \mathrm{H} 4) ; 7.64$ $(1 \mathrm{H} ; \mathrm{m} ; \mathrm{H} 5) ; 6.82(\mathrm{~d} ; J=7.6)$ and $6.74(\mathrm{~d} ; J=8.3),(1 \mathrm{H}$; $\mathrm{NHCH} ;(E / Z)$-isomer $) ; 4.98(\mathrm{~m})$ and $4.81(\mathrm{t} ; J=6.1),(1 \mathrm{H}$; $\mathrm{OH}$; $(E / Z)$-isomer); 4.98 and $4.05(1 \mathrm{H} ; \mathrm{m}$; $\mathrm{CH}$; $(E / Z)-$ isomer); 3.75-3.55 (2H; m; $\left.\mathrm{CH}_{2} \mathrm{OH}\right) ; 1.38\left(9 \mathrm{H} ; \mathrm{s} ;\left(\mathrm{CH}_{3}\right)_{3} \mathrm{C}\right)$. ${ }^{13} \mathrm{C}-\mathrm{NMR}\left(100 \mathrm{MHz}, \mathrm{DMSO}-d_{6}\right) \delta(\mathrm{ppm}): 171.9 ; 167.6$; $155.3 ; 155.2 ; 144.5 ; 140.9 ; 135.6 ; 135.5 ; 133.1 ; 132.9$; $131.2 ; 130.9 ; 130.7,130.1 ; 130.0 ; 118.4 ; 112.0 ; 78.3 ; 78.0$; 61.6; 61.2; 56.0; 54.0; 28.2. IR $\left(\mathrm{cm}^{-1} ; \mathrm{KBr}\right) v_{\max } 3346 ; 1685$; 2234. MS/ESI: [M-H]: 331.3

Tert-butyl (1S)-2-[(2E/Z)-2-(4-cyanobenzylidene)hydrazino]-1-(hydroxymethyl)-2-oxoethylcarbamate $9 f$

Yield: $70 \%$. m.p. $161^{0} \mathrm{C} .{ }^{1} \mathrm{H}-\mathrm{NMR}\left(500 \mathrm{MHz}, \mathrm{DMSO}-d_{6}\right)$ $\delta(\mathrm{ppm}): 11.71$ and $11.60(1 \mathrm{H} ; \mathrm{s} ; \mathrm{NHN}$; $(E / Z)$-isomer); 8.29 and $8.02(1 \mathrm{H} ; \mathrm{s} ; \mathrm{N}=\mathrm{CH}$; $(E / Z)$-isomer $) ; 7.90-7.80(4 \mathrm{H} ; \mathrm{m}$; $\mathrm{H} 2, \mathrm{H} 3, \mathrm{H} 5$ and $\mathrm{H} 6) ; 6.84(\mathrm{~d} ; J=7.4)$ and $6.73(\mathrm{~d} ; J=8.3)$, $(1 \mathrm{H} ; \mathrm{NHCH} ;(E / Z)$-isomer); $4.95(\mathrm{~m})$ and $4.82(\mathrm{t} ; J=6.4)$, $(1 \mathrm{H} ; \mathrm{OH} ;(E / Z)$-isomer); 4.95 and $4.06(1 \mathrm{H} ; \mathrm{m} ; \mathrm{CH} ;(E / Z)-$ isomer); 3.70-3.55 (2H; m; $\left.\mathrm{CH}_{2} \mathrm{OH}\right) ; 1.38\left(9 \mathrm{H}, \mathrm{s} ;\left(\mathrm{CH}_{3}\right)_{3} \mathrm{C}\right)$. ${ }^{13} \mathrm{C}-\mathrm{NMR}\left(125 \mathrm{MHz}, \mathrm{DMSO}-d_{6}\right) \delta(\mathrm{ppm}): 172.0 ; 167.7$; $155.3 ; 155.2 ; 144.8 ; 141.2 ; 138.8 ; 138.6 ; 132.7 ; 127.6$; $127.4 ; 118.7 ; 118.6 ; 111.8 ; 111.6 ; 78.3 ; 78.1 ; 61.5 ; 61.2$;
56.0; 54.0; 28.2. IR $\left(\mathrm{cm}^{-1} ; \mathrm{KBr}\right) v_{\max } 3375 ; 1678 ; 2228$. MS/ESI: [M-H]: 331.3

Tert-butyl (1S)-2-[(2E)-2-(2-hydroxybenzylidene)hydrazino]-1-(hydroxymethyl)-2-oxoethylcarbamate $\mathbf{9 g}$

Yield: $76 \%$. m.p. $183^{0} \mathrm{C} .{ }^{1} \mathrm{H}-\mathrm{NMR}\left(500 \mathrm{MHz}\right.$, DMSO- $\left.d_{6}\right)$ $\delta(\mathrm{ppm}): 11.70(1 \mathrm{H} ; \mathrm{s} ; \mathrm{NHN}) ; 11.13(1 \mathrm{H} ; \mathrm{s} ; \mathrm{Ph}-\mathrm{OH}) ; 8.44$ $(1 \mathrm{H} ; \mathrm{s} ; \mathrm{N}=\mathrm{CH},(E)$-isomer $) ; 7.50(1 \mathrm{H} ; \mathrm{d} ; J=7.4 ; \mathrm{H} 6) ; 7.28$ $(1 \mathrm{H} ; \mathrm{m} ; \mathrm{H} 4$ or $\mathrm{H} 5)$; $6.93-6.86(2 \mathrm{H} ; \mathrm{m} ; \mathrm{H} 3$ and $(\mathrm{H} 4$ or $\mathrm{H} 5))$; $6.84(1 \mathrm{H} ; \mathrm{d} ; J=7.8 ; \mathrm{NHCH}) ; 4.99(1 \mathrm{H} ; \mathrm{s} ; \mathrm{OH}) ; 4.05(1 \mathrm{H} ; \mathrm{m}$; $\mathrm{CH})$; 3.70-3.55 (2H; m; $\left.\mathrm{CH}_{2} \mathrm{OH}\right) ; 1.39\left(9 \mathrm{H} ; \mathrm{s} ;\left(\mathrm{CH}_{3}\right)_{3} \mathrm{C}\right)$. ${ }^{13} \mathrm{C}-\mathrm{NMR}\left(125 \mathrm{MHz}, \mathrm{DMSO}-d_{6}\right) \delta(\mathrm{ppm}): 171.8 ; 157.8$; $156.8 ; 141.2 ; 131.8 ; 129.8 ; 119.9 ; 119.1 ; 116.6 ; 78.8 ; 61.6$; 56.4; 28.6. IR $\left(\mathrm{cm}^{-1} ; \mathrm{KBr}\right) v_{\max } 3366 ; 1692 ; 1678$. MS/ESI: [M-H]: 322.3

Tert-butyl (1S)-2-[(2E)-2-(2,3-dihydroxybenzylidene) hydrazino]-1-(hydroxymethyl)-2-oxoethylcarbamate $\mathbf{9 h}$

Yield: $85 \%$. m.p. $176^{0} \mathrm{C} .{ }^{1} \mathrm{H}-\mathrm{NMR}\left(500 \mathrm{MHz}\right.$, DMSO- $\left.d_{6}\right)$ $\delta(\mathrm{ppm}): 11.70(1 \mathrm{H} ; \mathrm{s} ; \mathrm{NHN}) ; 10.96(1 \mathrm{H} ; \mathrm{s} ; \mathrm{C} 1-\mathrm{OH}$ or $\mathrm{C} 2-\mathrm{OH}) ; 9.19(1 \mathrm{H}$; s; $\mathrm{C} 1-\mathrm{OH}$ or $\mathrm{C} 2-\mathrm{OH}) ; 8.40(1 \mathrm{H}$; s; $\mathrm{N}=\mathrm{CH} ;(E)$-isomer $) ; 6.93(1 \mathrm{H} ; \mathrm{d} ; J=7.8 ; \mathrm{H} 6) ; 6.90-6.80(1 \mathrm{H}$; $\mathrm{m} ; \mathrm{H} 4) ; 6.90-6.80(1 \mathrm{H} ; \mathrm{m} ; \mathrm{NHCH}) ; 6.72(1 \mathrm{H} ; \mathrm{t} ; J=7.8 ; \mathrm{H} 5)$; $4.99(1 \mathrm{H} ; \mathrm{s} ; \mathrm{OH}) ; 4.05(1 \mathrm{H} ; \mathrm{m} ; \mathrm{CH}) ; 3.70-3.55(2 \mathrm{H} ; \mathrm{m}$; $\left.\mathrm{CH}_{2} \mathrm{OH}\right) ; 1.39\left(9 \mathrm{H} ; \mathrm{s} ;\left(\mathrm{CH}_{3}\right)_{3} \mathrm{C}\right) .{ }^{13} \mathrm{C}-\mathrm{NMR} \quad(125 \mathrm{MHz}$, DMSO- $\left.d_{6}\right) \delta(\mathrm{ppm}): 171.3 ; 155.2 ; 145.9 ; 145.5 ; 141.5$; $120.7 ; 119.1 ; 117.3 ; 116.5 ; 78.1 ; 61.5 ; 53.9 ; 28.2$. IR $\left(\mathrm{cm}^{-1}\right.$; KBr) $v_{\max } 3350 ; 1680 . \mathrm{MS} / \mathrm{ESI}:[\mathrm{M}-\mathrm{H}]: 338.3$

Tert-butyl (1S)-2-[(2E)-2-(2,4-dihydroxybenzylidene) hydrazino]-1-(hydroxymethyl)-2-oxoethylcarbamate $\mathbf{9 i}$

Yield: $74 \%$. m.p. $150^{0} \mathrm{C} .{ }^{1} \mathrm{H}-\mathrm{NMR}\left(500 \mathrm{MHz}\right.$, DMSO- $\left.d_{6}\right)$ $\delta(\mathrm{ppm}): 11.50(1 \mathrm{H} ; \mathrm{s} ; \mathrm{NHN}) ; 11.30(1 \mathrm{H} ; \mathrm{s} ; \mathrm{C} 1-\mathrm{OH}$ or $\mathrm{C} 3-\mathrm{OH})$; $9.92(1 \mathrm{H}$; s; $\mathrm{C} 1-\mathrm{OH}$ ou $\mathrm{C} 3-\mathrm{OH}) ; 8.30(1 \mathrm{H}$; s; $\mathrm{N}=\mathrm{CH} ;(E)$-isomer $) ; 7.26(1 \mathrm{H} ; \mathrm{d} ; J=8.4 ; \mathrm{H} 6) ; 6.80(1 \mathrm{H} ; \mathrm{d}$; $J=7.7 ; \mathrm{NHCH}) ; 6.35-6.30(1 \mathrm{H} ; \mathrm{m} ; \mathrm{H} 5) ; 6.29(1 \mathrm{H} ; \mathrm{s} ; \mathrm{H} 3)$; $4.95(1 \mathrm{H} ; \mathrm{s} ; \mathrm{OH}) ; 4.02(1 \mathrm{H} ; \mathrm{m} ; \mathrm{CH}) ; 3.70-3.50(2 \mathrm{H} ; \mathrm{m}$; $\left.\mathrm{C}_{2} \mathrm{OH}\right) ; 1.39\left(9 \mathrm{H} ; \mathrm{s} ;\left(\mathrm{CH}_{3}\right)_{3} \mathrm{C}\right) .{ }^{13} \mathrm{C}-\mathrm{NMR} \quad(125 \mathrm{MHz}$, DMSO- $\left.d_{6}\right) \delta$ (ppm): $170.8 ; 160.3 ; 157.9 ; 155.2 ; 141.8$; $128.0 ; 110.4 ; 107.6 ; 102.3 ; 78.0 ; 61.1 ; 53.9 ; 28.2$. IR $\left(\mathrm{cm}^{-1}\right.$; $\mathrm{KBr}) v_{\max } 3200 ; 1678$. MS/ESI: [M-H]: 338.3

Tert-butyl (1S)-2-[(2E)-2-(2,5-dihydroxybenzylidene) hydrazino]-1-(hydroxymethyl)-2-oxoethylcarbamate $\mathbf{9 j}$

Yield: $79 \%$. m.p. $148^{0} \mathrm{C} .{ }^{1} \mathrm{H}-\mathrm{NMR}\left(500 \mathrm{MHz}, \mathrm{DMSO}-d_{6}\right)$ $\delta(\mathrm{ppm}): 11.58(1 \mathrm{H} ; \mathrm{s} ; \mathrm{NHN}) ; 10.24(1 \mathrm{H} ; \mathrm{s} ; \mathrm{C} 1-\mathrm{OH}$ or $\mathrm{C} 4-\mathrm{OH}) ; 8.95(1 \mathrm{H} ; \mathrm{s} ; \mathrm{C} 1-\mathrm{OH}$ or $\mathrm{C} 4-\mathrm{OH}) ; 8.36(1 \mathrm{H} ; \mathrm{s}$; $\mathrm{N}=\mathrm{CH} ;(E)$-isomer $) ; 6.92(1 \mathrm{H} ; \mathrm{d} ; J=2.0 ; \mathrm{H} 6) ; 6.81(1 \mathrm{H} ; \mathrm{d}$; $J=7.8 ; \mathrm{NHCH}) ; 6.75-6.65(2 \mathrm{H} ; \mathrm{m} ; \mathrm{H} 3$ and $\mathrm{H} 4) ; 4.97(1 \mathrm{H}$; $\mathrm{m} ; \mathrm{OH}) ; 4.02(1 \mathrm{H} ; \mathrm{m} ; \mathrm{CH}) ; 3.70-3.50\left(2 \mathrm{H} ; \mathrm{m} ; \mathrm{CH}_{2} \mathrm{OH}\right) ; 1.39$ $\left(9 \mathrm{H} ; \mathrm{s} ;\left(\mathrm{CH}_{3}\right)_{3} \mathrm{C}\right) .{ }^{13} \mathrm{C}-\mathrm{NMR}\left(125 \mathrm{MHz}, \mathrm{DMSO}-d_{6}\right) \delta(\mathrm{ppm})$ : $171.7 ; 155.7 ; 150.4 ; 149.8 ; 141.2 ; 120.9 ; 119.1 ; 117.5$; 111.5; 78.6; 61.7; 54.4; 28.6. IR $\left(\mathrm{cm}^{-1} ; \mathrm{KBr}\right) v_{\max } 3358$; 1678. MS/ESI: [M-H]: 338.3

Tert-butyl (1S)-2-[(2E)-2-(2-hydroxy-3-methoxybenzylidene)hydrazino]-1-(hydroxymethyl)-2-oxoethylcarbamate 9k

Yield: $80 \%$. m.p.: $125^{0} \mathrm{C} .{ }^{1} \mathrm{H}-\mathrm{NMR}\left(500 \mathrm{MHz}\right.$, DMSO- $\left.d_{6}\right)$ $\delta(\mathrm{ppm}): 11.69(1 \mathrm{H} ; \mathrm{s} ; \mathrm{NHN}) ; 10.81(1 \mathrm{H} ; \mathrm{s} ; \mathrm{C} 1-\mathrm{OH}) ; 8.45$ $(1 \mathrm{H} ; \mathrm{s} ; \mathrm{N}=\mathrm{CH} ;(E)$-isomer); $7.10(1 \mathrm{H} ; \mathrm{dd} ; J=7.8$ and $J=1.0$; H6); $7.01(1 \mathrm{H}$; dd; $J=8.0$ and $J=1.0 ; \mathrm{H} 4) ; 6.90-6.78(2 \mathrm{H}$; m; H5 and $\mathrm{NHCH}) ; 4.99(1 \mathrm{H} ; \mathrm{s} ; \mathrm{OH}) ; 4.04(1 \mathrm{H} ; \mathrm{m} ; \mathrm{CH})$; 
$3.80\left(3 \mathrm{H} ; \mathrm{s} ; \mathrm{CH}_{3} \mathrm{O}\right) ; 3.70-3.55\left(2 \mathrm{H} ; \mathrm{m} ; \mathrm{C}_{2} \mathrm{OH}\right) ; 1.39(9 \mathrm{H} ; \mathrm{s}$; $\left.\left(\mathrm{CH}_{3}\right)_{3} \mathrm{C}\right) \cdot{ }^{13} \mathrm{C}-\mathrm{NMR}\left(125 \mathrm{MHz}\right.$, DMSO- $\left.d_{6}\right) \delta(\mathrm{ppm}): 171.3$; $155.2 ; 147.9 ; 145.9 ; 140.5 ; 120.7 ; 119.0 ; 117.6,112.8 ; 78.1$; $61.2 ; 55.8 ; 54.0 ; 28.2$.

\section{IR $\left(\mathrm{cm}^{-1} ; \mathrm{KBr}\right) v_{\max } 3358 ; 1684$. MS/ESI: [M-H] $: 352.4$}

Tert-butyl (1S)-2-[(2E)-2-(3,4-dihydroxybenzylidene) hydrazino]-1-(hydroxymethyl)-2-oxoethylcarbamate 91

Yield: $85 \%$. m.p. $138^{0} \mathrm{C} .{ }^{1} \mathrm{H}-\mathrm{NMR}\left(500 \mathrm{MHz}\right.$, DMSO- $\left.d_{6}\right)$ $\delta(\mathrm{ppm}): 11.20(1 \mathrm{H} ; \mathrm{s} ; \mathrm{NHN}) ; 9.40(1 \mathrm{H} ; \mathrm{s} ; \mathrm{C} 2-\mathrm{OH}$ or $\mathrm{C} 3-\mathrm{OH}) ; 9.26(1 \mathrm{H} ; \mathrm{s} ; \mathrm{C} 2-\mathrm{OH}$ or $\mathrm{C} 3-\mathrm{OH}) ; 8.03(1 \mathrm{H} ; \mathrm{s}$; $\mathrm{N}=\mathrm{CH} ;(E)$-isomer); $7.17(1 \mathrm{H} ; \mathrm{d} ; J=1.5 ; \mathrm{H} 2) ; 6.90(1 \mathrm{H} ; \mathrm{s}$; H6); $6.77(1 \mathrm{H} ; \mathrm{s} ; \mathrm{H} 5) ; 6.73(1 \mathrm{H} ; \mathrm{d} ; J=8.3 ; \mathrm{NHCH}) ; 4.95$ $(1 \mathrm{H} ; \mathrm{m} ; \mathrm{OH}) ; 4.01(1 \mathrm{H} ; \mathrm{m} ; \mathrm{CH}) ; 3.70-3.50\left(2 \mathrm{H} ; \mathrm{m} ; \mathrm{CH}_{2} \mathrm{OH}\right)$; $1.39\left(9 \mathrm{H} ; \mathrm{s} ;\left(\mathrm{CH}_{3}\right)_{3} \mathrm{C}\right) .{ }^{13} \mathrm{C}-\mathrm{NMR}\left(125 \mathrm{MHz}, \mathrm{DMSO}-d_{6}\right) \delta$ (ppm): 171.6; 155.7; 148.2; 147.7; 144.3; 129.1; 120.5; $116.0 ; 113.0 ; 78.6 ; 62.2 ; 54.5 ; 28.6$. IR $\left(\mathrm{cm}^{-1} ; \mathrm{KBr}\right) v_{\max }$ 3281; 1672. MS/ESI: [M-H]: 338.3

Tert-butyl (1S)-2-[(2E)-2-(3,4-dimethoxybenzylidene) hydrazino]-1-(hydroxymethyl)-2-oxoethylcarbamate $9 \mathbf{m}$

Yield: $82 \%$. m.p. $120^{0} \mathrm{C} .{ }^{1} \mathrm{H}-\mathrm{NMR}\left(500 \mathrm{MHz}, \mathrm{DMSO}-d_{6}\right)$ $\delta(\mathrm{ppm}): 11.32(1 \mathrm{H} ; \mathrm{s} ; \mathrm{NHN}) ; 8.14(1 \mathrm{H} ; \mathrm{s} ; \mathrm{N}=\mathrm{CH} ;(E)-$ isomer); $7.29(1 \mathrm{H} ; \mathrm{s} ; \mathrm{H} 2) ; 7.17(1 \mathrm{H} ; \mathrm{m} ; \mathrm{H} 6) ; 7.00(1 \mathrm{H} ; \mathrm{m}$; $\mathrm{H} 5) ; 6.76(1 \mathrm{H} ; \mathrm{d} ; J=7.3 ; \mathrm{N} \underline{\mathrm{HCH}}) ; 4.93(1 \mathrm{H} ; \mathrm{m} ; \mathrm{OH}) ; 4.02$ $(1 \mathrm{H} ; \mathrm{m} ; \mathrm{CH}) ; 3.80\left(6 \mathrm{H} ; \mathrm{s} ; \mathrm{C}_{2}-\mathrm{OCH}_{3}\right.$ and $\left.\mathrm{C} 3-\mathrm{OCH}_{3}\right) ; 3.75-$ $3.55\left(2 \mathrm{H} ; \mathrm{m} ; \mathrm{CH}_{2} \mathrm{OH}\right) ; 1.39\left(9 \mathrm{H} ; \mathrm{s} ;\left(\mathrm{CH}_{3}\right)_{3} \mathrm{C}\right) .{ }^{13} \mathrm{C}-\mathrm{NMR}$ $\left(125 \mathrm{MHz}, \mathrm{DMSO}-d_{6}\right) \delta(\mathrm{ppm}): 171.8 ; 155.7 ; 150.9 ; 149.5$; $143.6 ; 127.4 ; 121.4 ; 111.9 ; 108.7 ; 78.5 ; 61.6 ; 56.0 ; 55.9$; 54.6; 28.6. IR $\left(\mathrm{cm}^{-1} ; \mathrm{KBr}\right): 3227(\mathrm{O}-\mathrm{H}) ; 1671(\mathrm{COCH}$ and COO). MS/ESI: [M-H]: 366.4

Tert-butyl (1S)-2-[(2E)-2-(2-hydroxy-4-methoxybenzylidene)hydrazino]-1-(hydroxymethyl)-2-oxoethylcarbamate 9n

Yield: $84 \%$. m.p. $182^{\circ} \mathrm{C} .{ }^{1} \mathrm{H}-\mathrm{NMR}\left(500 \mathrm{MHz}, \mathrm{DMSO}-d_{6}\right)$ $\delta(\mathrm{ppm}): 11.60(1 \mathrm{H} ; \mathrm{s} ; \mathrm{NHN}) ; 11.46(1 \mathrm{H} ; \mathrm{s} ; \mathrm{C} 1-\mathrm{OH}) ; 8.35$ $(1 \mathrm{H} ; \mathrm{s} ; \mathrm{N}=\mathrm{CH} ;(E)$-isomer $) ; 7.39(1 \mathrm{H} ; \mathrm{d} ; J=8.4 ; \mathrm{H} 6) ; 6.84$ $(1 \mathrm{H} ; \mathrm{d} ; J=7.4 ; \mathrm{NHCH}) ; 6.55-6.40(2 \mathrm{H} ; \mathrm{m} ; \mathrm{H} 3$ and $\mathrm{H} 5) ; 4.98$ $(1 \mathrm{H} ; \mathrm{m} ; \mathrm{OH}) ; 4.02(1 \mathrm{H} ; \mathrm{m} ; \mathrm{CH}) ; 3.76\left(3 \mathrm{H} ; \mathrm{s} ; \mathrm{CH}_{3} \mathrm{O}\right) ; 3.70-$ $3.50\left(2 \mathrm{H} ; \mathrm{m} ; \mathrm{CH}_{2} \mathrm{OH}\right) ; 1.39\left(9 \mathrm{H} ; \mathrm{s} ;\left(\mathrm{CH}_{3}\right)_{3} \mathrm{C}\right) .{ }^{13} \mathrm{C}-\mathrm{NMR}$ $\left(125 \mathrm{MHz}, \mathrm{DMSO}-d_{6}\right) \delta(\mathrm{ppm}): 171.5 ; 162.5 ; 158.3 ; 155.7$; $141.8 ; 128.3 ; 112.1 ; 106.9 ; 101.4 ; 78.5 ; 61.6 ; 56.3 ; 55.6$; 28.6. IR $\left(\mathrm{cm}^{-1} ; \mathrm{KBr}\right) v_{\max } 3375 ; 1677$. MS/ESI: [M-H] : 352.4

Tert-butyl (1S)-1-(hydroxymethyl)-2-[(2E)-2-(2methoxybenzylidene)hydrazino]-2-oxoethylcarbamate 9o

Yield: $75 \%$. m.p. $115^{0} \mathrm{C} .{ }^{1} \mathrm{H}-\mathrm{NMR}(500 \mathrm{MHz}$, DMSO-d6) $\delta(\mathrm{ppm}): 11.46(1 \mathrm{H} ; \mathrm{s} ; \mathrm{NHN}) ; 8.58(1 \mathrm{H} ; \mathrm{s} ; \mathrm{N}=\mathrm{CH} ;(E)-$ isomer); $7.82(1 \mathrm{H} ; \mathrm{d} ; \mathrm{J}=7.8 ; \mathrm{H} 5) ; 7.39(1 \mathrm{H} ; \mathrm{m} ; \mathrm{H} 3) ; 7.10$ $(1 \mathrm{H} ; \mathrm{s} ; \mathrm{H} 2) ; 7.00(1 \mathrm{H} ; \mathrm{m} ; \mathrm{H} 4) ; 6.75(1 \mathrm{H} ; \mathrm{d} ; \mathrm{J}=7.8 ; \mathrm{NHCH})$; $4.94(1 \mathrm{H} ; \mathrm{m} ; \mathrm{OH}) ; 4.94(1 \mathrm{H} ; \mathrm{m} ; \mathrm{CH}) ; 3.86\left(3 \mathrm{H} ; \mathrm{s} ; \mathrm{CH}_{3} \mathrm{O}\right)$; 3.75-3.55 (2H; m; $\left.\mathrm{CH}_{2} \mathrm{OH}\right) ; 1.39(9 \mathrm{H}$; s; $(\mathrm{CH} 3) 3 \mathrm{C}-) .{ }^{13} \mathrm{C}-$ NMR $(125 \mathrm{MHz}$, DMSO-d6) $\delta(\mathrm{ppm}): 171.9 ; 158.2 ; 155.7$; $142.8 ; 132.0 ; 125.9 ; 122.7 ; 121.2 ; 112.2 ; 78.7 ; 62.1 ; 56.5$; 56.1; 28.6. IR (cm-1; KBr) $v_{\max } 3321 ; 1670$; . MS/ESI: [M$\mathrm{H}]: 336.3$

(4S)- $N$ '-[(E)-(3-nitrophenyl)methylidene]-2-oxo-1,3oxazolidine-4-carbohydrazide 10a

Yield: $55 \%$. m.p. $190^{0} \mathrm{C} .{ }^{1} \mathrm{H}-\mathrm{NMR}\left(500 \mathrm{MHz}, \mathrm{DMSO}-\mathrm{d}_{6}\right)$ $\delta(\mathrm{ppm}): 11.91(1 \mathrm{H} ; \mathrm{s} ;-\mathrm{NHN}=) ; 8.47(1 \mathrm{H} ; \mathrm{m} ; \mathrm{H} 1) ; 8.12$ $(1 \mathrm{H} ; \mathrm{s} ;-\mathrm{N}=\mathrm{CH}-;(E)$-isomer $) ; 8.25(1 \mathrm{H} ; \mathrm{m} ; \mathrm{H} 3$ or $\mathrm{H} 5) ; 8.16$
$(1 \mathrm{H} ; \mathrm{m} ; \mathrm{H} 3$ or $\mathrm{H} 5) ; 8.04(1 \mathrm{H} ; \mathrm{s} ; \mathrm{NHCH}) ; 7.75-7.65(1 \mathrm{H} ; \mathrm{m}$; $\mathrm{H} 4) ; 5.11(1 \mathrm{H} ;$; dd; $\mathrm{J}=12.0$ and $\mathrm{J}=6.4 ; \mathrm{CH}) ;(1 \mathrm{H} ; \mathrm{t} ; \mathrm{J}=12.0$; $\left.\mathrm{CHH}^{\prime}\right) ; \quad 4.35-4.25\left(1 \mathrm{H} ; \mathrm{m}\right.$; CHH'). ${ }^{13} \mathrm{C}-\mathrm{NMR}(125 \mathrm{MHz}$, DMSO-d $\left._{6}\right) \delta(\mathrm{ppm}): 171.6 ; 158.8 ; 148.2 ; 145.8 ; 135.8$; $133.3 ; 130.5 ; 124.5 ; 121.6 ; 66.8 ; 53.3$. IR (cm-1; KBr) $v_{\max }$ 1767; 1689. MS/ESI: [M-H]: 277.3

(4S)- $N$ '-[(E)- (3-cyanophenyl)methylidene]-2-oxo-1,3oxazolidine-4-carbohydrazide $\mathbf{1 0 b}$

Yield: $50 \%$. m.p. $195^{0} \mathrm{C} .{ }^{1} \mathrm{H}-\mathrm{NMR}\left(500 \mathrm{MHz}\right.$, DMSO- $\left.d_{6}\right)$ $\delta(\mathrm{ppm}): 11.84(1 \mathrm{H} ; \mathrm{s} ; \mathrm{NHN}) ; 8,02(1 \mathrm{H} ; \mathrm{s} ; \mathrm{N}=\mathrm{CH} ;(E)-$ isomer); 8.20-7.95 $(3 \mathrm{H} ; \mathrm{m} ; \mathrm{H} 1, \mathrm{H} 5$ and $\mathrm{NHCH}) ; 7.86(1 \mathrm{H} ; \mathrm{d}$; $J=7.8 ; \mathrm{H} 3) ; 7.64(1 \mathrm{H} ; \mathrm{m} ; \mathrm{H} 4) ; 5.11(1 \mathrm{H} ; \mathrm{dd} ; J=9.8$ and $J=$ $4.9 ; \mathrm{CH}) ; 4.71\left(1 \mathrm{H} ; \mathrm{t} ; J=9,8 \mathrm{CHH}^{\prime}\right) ; 4.28\left(1 \mathrm{H} ; \mathrm{m} ; \mathrm{CHH}^{\prime}\right)$. ${ }^{13} \mathrm{C}-\mathrm{NMR} \quad\left(100 \mathrm{MHz}, \mathrm{DMSO}-d_{6}\right) \delta(\mathrm{ppm}): 171.7 \quad 159.0$; $146.0 ; 135.3,133.5,131.6 ; 130.2 ; 118.5 ; 112.0 ; 66.9 ; 53.3$. IR $\left(\mathrm{cm}^{-1} ; \mathrm{KBr}\right) v_{\max } 2227 ; 1746 ; 1694$. MS/ESI: [M-H]: 257.3

\section{REFERENCES}

[1] Organização Mundial da Saúde, Tuberculosis MDR-TB \& XDRTB 2010 report, http://www.who.int/tb/features_archive/ world_tb_day_2010/mdrfactsheet15mar10_19h00.pdf (Accessed December 15, 2010)

[2] De Souza, M.V.N. Promising candidates in clinical trials against multidrug-resistant tuberculosis (MDR-TB) based on natural products. Fitoterapia, 2009, 80, 453-60.

[3] De Souza, M.V.N.; Ferreira, M.L.; Pinheiro A.C.; Saraiva, M.F. Almeida, M.V.; Valle, M.S. Synthesis and Biological Aspects of Mycolic Acids: An Important Target Against Mycobacterium tuberculosis. Scientific WorldJournal, 2008, 8, 720-51.

[4] De Souza, M.V.N. Promising Drugs Against Tuberculosis. Recent Pat. Antiinfect. Drug Discov., 2006, 1, 33-45.

[5] De Souza, M.V.N. Current Status and Future Prospects for New Therapies for Pulmonary Tuberculosis. Curr. Opin. Pulm. Med., 2006, 12, 167-71.

[6] Gordeev, M.F.; Luehr, G.W.; Hui, H.C.; Gordon, E.M.; Patel, D.V. Combinatorial Chemistry of Natural Products: Solid Phase Synthesis of D- and L-Cycloserine Derivatives. Tetrahedron, 1998, 54 15879-90.

[7] Petri Jr., W.A. In The Pharmacological basis of Therapeutics; Goodman \& Gilman'; Mc Graw Hill: New York, 2001; pp.127394.

[8] Lester, W. In Pulmonary Diseases and Disorders; Fishman, A.P.; Mc Graw Hill: New York, 1998; pp.1305-23.

[9] Fenn, T.D.; Stamper, G.F.; Morollo, A.A.; Ringe, D. A Side Reaction of Alanine Racemase: Transamination of Cycloserine. Biochemistry, 2003, 42, 5775-83.

[10] Chung, S.; Jonhson, M.S.; Gronenborn, A.M. L-Cycloserine: A Potent Anticonvulsant. Epilepsia, 1984, 25(3), 353-62.

[11] Rollas, S.; Küçükgüzel, G. Biological activities of hydrazones derivatives. Molecules, 2007, 12, 1910-39.

[12] Ferreira, M.L.; Gonçalves, R.S.B.; Cardoso, L.N.F.; Kaiser, C.R.; Candéa, A.L.P.; Henriques, M.G.M.O.; Lourenço, M.C.; Bezerra, F.A.F.M.; De Souza, M.V.N. Synthesis and Antitubercular Activity of Heteroaromatic Isonicotinoyl and 7-Chloro-4-Quinolinyl Hydrazone Derivatives. Scientific WorldJournal, 2010, 10, 1347-55.

[13] Ferreira, M.L., Candéa, A.L.P., Henriques, M.G.M.O., Kaiser, C.R., Lima, C.H.S., De Souza, M.V.N. Synthesis and Cytotoxic Evaluation of Disubstituted $N$-Acylhydrazones Pyrazinecarbohydrazide Derivatives. Lett. Drug Des. Discov., 2010, 7, 275 - 80.

[14] Candéa, A.L.P., Ferreira, M.L., Pais, K.C., Cardoso, L.N.F., Kaiser, C.R., Henriques, M.G.M.O., Lourenço, M.C.S., Bezerra, F.A.F.M., De Souza, M.V.N. Synthesis and antitubercular activity of 7-chloro-4-quinolinylhydrazones derivatives. Bioorg. Med. Chem. Lett., 2009, 19, 6272 - 74.

[15] Vergara, F.M.F.; Lima, C.H.S.; Henriques, M.G.M.O.; Candéa, A.L.P.; Lourenço, M.C.S.; Ferreira, M.L.; Kaiser, C.R.; De Souza, M.V.N. Synthesis and antimycobacterial activity of $N^{\prime}-[(E)$ 
(monosubstituted-benzylidene)]-2-pyrazinecarbohydrazide derivatives. Eur. J. Med. Chem., 2009, 44, 4954-59.

[16] Pinheiro, A.C.; De Souza, M.V.N.; Tiekink, E.R.T.; Wardell, J.L.; Wardell, S.M.S.V. Benzyl N-((S)-2-hydroxy-1-\{N'-[(E)-2methoxybenzylidene] hydrazinecarbonyl \}ethyl)carbamate from synchrotron data. Acta Crystallogr., Sect. E: Struct. Rep. Online, 2010, 66, 1004-05.
[17] De Souza, M.V.N.; Pinheiro, A.C.; Tiekink, E.R.T., Wardell, S.M.S.V.; Wardell, J.L. Benzyl N-[(S)-2-hydroxy-1-(\{[(E)-2hydroxy-4-methoxybenzylidene] drazinyl\}carbonyl)ethyl]carbamate. Acta Crystallogr., Sect. E: Struct. Rep. Online, 2010, 66, 3253-54.

[18] De Souza, M.V.N.; Pinheiro, A.C.; Tiekink, E.R.T.; Wardell, S.M.S.V.; Wardell, J.L. Benzyl N-\{(1S)-2-hydroxy-1-[N'-(2nitrobenzylidene)hydrazinylcarbonyl] ethyl $\}$ carbamate. Acta Crystallogr., Sect. E: Struct. Rep. Online, 2010, 66, 2023-24. 